ESAIM: M2AN 50 (2016) 1371-1401

DOI: $10.1051 / \mathrm{m} 2 \mathrm{an} / 2015076$
ESAIM: Mathematical Modelling and Numerical Analysis

www.esaim-m2an.org

\title{
NUMERICAL ASPECTS OF LARGE-TIME OPTIMAL CONTROL OF BURGERS EQUATION
}

\author{
Navid Allahverdi ${ }^{1,2}$, Alejandro Pozo ${ }^{2}$ and Enrique Zuazua ${ }^{3,2}$
}

\begin{abstract}
In this paper, we discuss the efficiency of various numerical methods for the inverse design of the Burgers equation, both in the viscous and in the inviscid case, in long time-horizons. Roughly, the problem consists in, given a final desired target, to identify the initial datum that leads to it along the Burgers dynamics. This constitutes an ill-posed backward problem. We highlight the importance of employing a proper discretization scheme in the numerical approximation of the equation under consideration to obtain an accurate approximation of the optimal control problem. Convergence in the classical sense of numerical analysis does not suffice since numerical schemes can alter the dynamics of the underlying continuous system in long time intervals. As we shall see, this may end up affecting the efficiency on the numerical approximation of the inverse design, that could be polluted by spurious high frequency numerical oscillations. To illustrate this, two well-known numerical schemes are employed: the modified Lax-Friedrichs scheme (MLF) and the Engquist-Osher (EO) one. It is by now wellknown that the MLF scheme, as time tends to infinity, leads to asymptotic profiles with an excess of viscosity, while EO captures the correct asymptotic dynamics. We solve the inverse design problem by means of a gradient descent method and show that EO performs robustly, reaching efficiently a good approximation of the minimizer, while MLF shows a very strong sensitivity to the selection of cell and time-step sizes, due to excess of numerical viscosity. The achieved numerical results are confirmed by numerical experiments run with the open source nonlinear optimization package (IPOPT).
\end{abstract}

Mathematics Subject Classification. 49M, 35Q35, 65M06.

Received January 27, 2015. Revised October 3, 2015. Accepted October 13, 2015.

\section{INTRODUCTION}

We analyze the numerical approximation of the inverse design problem for the Burgers equation, both in the viscous and in the inviscid case:

$$
\begin{cases}\partial_{t} u+\partial_{x}\left(\frac{u^{2}}{2}\right)=\nu \partial_{x x} u, & x \in \mathbb{R}, t>0, \\ u(x, 0)=u_{0}(x), & x \in \mathbb{R} .\end{cases}
$$

Keywords and phrases. Burgers equation, inverse design, optimization, numerics, descent method.

1 New York City College of Technology. 300 Jay Street, NY 11201 Brooklyn, USA. nhajiallahverdipur@citytech.cuny.edu

2 BCAM - Basque Center for Applied Mathematics. Alameda Mazarredo 14, 48009 Bilbao, Basque Country, Spain.

alejandropozo@gmail.com

3 Departamento de Matemáticas, Universidad Autónoma de Madrid, Cantoblanco, 28049 Madrid, Spain.

enrique.zuazua@uam.es 
Given a time $T>0$ and a target function $u^{*}$ the aim is to identify the initial datum $u_{0}$ so that the solution, at time $t=T$, reaches the target $u^{*}$ or gets as close as possible to it. In particular, we focus on problems with large values of $T$, for which convergence of the numerical schemes in the classical sense of numerical analysis might not suffice to obtain accurate results.

Essentially, the question consists in solving the Burgers equation backwards, a problem that is ill-posed. In the viscous case $\nu>0$, this is due to the intrinsic strong time-irreversibility of the parabolic Burgers equation that is enhanced by the nonlinear phenomena governing the hyperbolic dynamics. In the inviscid hyperbolic case $\nu=0$, the nonlinearity of the model that produces, in particular, the emergence of shock discontinuities, makes the problem to be ill-posed too, having, in some cases, multiple solutions.

We formulate the problem from the point of view of optimal control. Using a least square approach, we consider the minimization of the following functional:

$$
\mathcal{J}\left(u_{0}\right)=\frac{1}{2} \int_{\mathbb{R}}\left(u(x, T)-u^{*}(x)\right)^{2} \mathrm{~d} x,
$$

where $u$ is the solution of the Burgers equation and the initial data $u_{0}$ lies in a suitable functional class, for instance $L^{1}(\mathbb{R}) \cap L^{\infty}(\mathbb{R})$.

This issue is motivated by the challenging problem of sonic-boom minimization for supersonic aircrafts [2]. The propagation of the sonic-boom in the atmosphere, perceived as loud noises on the ground, is governed by an augmented Burgers equation [12]. At present, in order to design the new generation of supersonic aircrafts, the objective is to minimize the loudness of the sonic-boom on the ground, with the control variable being the initial noise generated from the aircraft. The near field noise can be tailored via modifying the geometry of the aircraft. The travel time of the signal to the ground is larger than the time scale of the initial disturbance by orders of magnitude and this motivates our study of large time control of the sonic-boom propagation.

The optimal control problem above for the Burgers equation, a simplified version of the complete sonic-boom model, arises naturally. One of the key ingredients is that the time horizon under consideration $[0, T]$, for practical purposes, needs to be long. As we shall see, this makes the choice of the numerical scheme approximating the PDE to be a sensitive issue, since schemes that do not yield the correct long-time dynamics are incapable of providing an accurate approximation of the optimal control. We shall focus on this particular Burgers model. But similar issues arise in other contexts, involving different PDE, in many applications in physical sciences, engineering, economics and management disciplines. This is particularly the case in the context of data assimilation [16] with applications in climate forecasting and hydrology modeling, or in identification of pollution source problems [30].

The optimal control problem (2.1) has already been addressed by several authors in the past, both from the continuous and the discrete point of view, but not with emphasis on the effect of long time horizons as we do it in here and that, as we shall see, has significant effects. As we already pointed out, the interplay of discretization and optimization makes this issue really challenging.

In the past, special attention has been paid to inviscid equations and the effect that shock discontinuities may have in optimal control problems. In this direction, among others, $[4,6,27,36]$ address the issue of linearizing the system around solutions developing shock discontinuities. There has been also an extensive research regarding the corresponding adjoint system and its discretization (see, for instance, $[5,23,28]$ ). In $[19,20]$ the authors analyze the pointwise convergence of the linearized and adjoint approximations for discontinuous solutions in a discretize-then-optimize approach. In particular, they already point out the importance of controlling the diffusion in order to obtain convergence to the corresponding solutions of the systems. Our approach confirms this fact from a different perspective, showing that the addition of artificial viscosity to the system needs to be tuned carefully, since the efficiency of the optimization algorithms can be negatively affected in long time horizons.

Moreover, the interplay between the optimize-then-discretize and the discretize-then-optimize approaches was analyzed in [35], where the author shows the critical role of the One-Sided Lipschitz condition (OSLC) in the equivalence of both approaches. In the present paper we opt for the latter, which does not take into account 
the derivatives with respect to the location shocks. Nevertheless, our conclusions, concerning the performance in long time horizons, can be applied to the former too.

In our numerical experiments we avoid discontinuities by considering targets and time-horizons, so that the relevant solutions emerging in the optimization process do not have enough time to develop wavefronts. Thus, the sensitivity of the location of shocks does not play an important role in our experiments. In that way, we focus only on the large-time effect, which may remain hidden when shocks arise (see Rem. 4.1 for further details). Of course, this would be an additional issue to be taken into account in the challenging inverse design problem for the inviscid Burgers equation in the presence of shocks.

In this paper we consider both the viscous, $\nu>0$, and the inviscid case, $\nu=0$, of the Burgers equation. The first one leads to a non-linear parabolic problem while the second one constitutes a nonlinear hyperbolic conservation law. The solutions to the inviscid Burgers equation $(\nu=0)$ may develop shocks and, if $u_{0} \in L^{1}(\mathbb{R})$, as time tends to infinity, they converge towards a self-similar N-wave (cf. [32]). Even if the scaling is the same, this behavior differs significantly from the viscous version (1.1), which is of parabolic nature (see [25]). The self-similar profiles are then diffusive, smooth and of constant sign. Nevertheless, when $\nu$ is sufficiently small and time is large (but not enough for the viscosity to be dominant), the behavior of the solutions is close to the hyperbolic case [29].

At the numerical level, when solving (1.1) with usual finite-difference conservative schemes, this asymptotic dichotomy for large time needs to be handled carefully since, in particular, the excess of numerical viscosity may destroy the long-time hyperbolic dynamics to make it parabolic. This issue was carefully analyzed in [26] where it was shown that, while the large time behavior of the inviscid dynamics is correctly captured by means of the Engquist-Osher scheme, the Lax-Friedrichs scheme fails to do so, due to the excess of viscosity that destroys the $\mathrm{N}$-wave dynamics and leads to a viscous profile. This pathology may arise also for the viscous Burgers equation when the numerical viscosity dominates the physical one.

In this work, we emphasize the consequences of this fact at the level of inverse design. This is done by means of a gradient descent method, following a discretize-then-optimize strategy together with the adjoint methodology. We also use IPOPT, an open-source software package for nonlinear optimization [37], to support our results. Note, however, that the large-time behavior dichotomy can be extended to other methods.

Gradient descent methods, such as the steepest descent method or conjugate gradient method, are attractive due to their algorithm simplicity. Descent methods do not require calculating second order derivatives. This makes them be a suitable approach for optimizing large scale problems, where the cost of computing the Hessian matrix and solving the corresponding linear system becomes prohibitive. The rate of convergence in gradient methods is related to the condition number. Generally, as the condition number increases, the contours of the functional elongates in one direction and convergence degrades. Also, in the case of non-convex functionals, gradient descent provides sequences that exhibit highly oscillatory behaviors. Recently, however, a variety of new descent methods was developed, based on Lojasiewicz's inequality, which ensures convergence towards critical points without requiring the convexity of the functional $[1,3,24,33]$.

The functional $\mathcal{J}$ under consideration, due to the quadratic nonlinearity involved in the Burgers equation, fails to be convex or, at least, there is no evidence of its convexity. Consequently, the existence of several critical points cannot be excluded, in principle. However, in view of the recent literature mentioned above, because of the analytic dependence of solutions on initial data and the fact that in those circumstances the Łojaciewicz inequality is fulfilled, one expects the convergence of gradient descent methods towards local minimizers. This is something we observe in our numerical simulations, but the velocity of convergence turns out to depend significantly on the numerical scheme that is employed for approximating the PDE and the gradient descent method implemented. The main aim of this paper is to accurately describe the various phenomena that overlap when handling numerically this inverse design problem.

As we shall see, while the direct implementation of a discrete approach based on the Enguist-Osher discretization leads to rather robust and satisfactory results at the level of the inverse design, the results turn out to be very sensitive to the discretization parameters when employing the Lax-Friedrichs scheme and its modified version coping with viscosity. With the latter, in some instances, the recovered inverse profile appears 
to be corrupted by unwanted spurious numerical high frequency components. Our numerical results are also tested and compared with those obtained with the optimization package IPOPT to confirm our predictions and conclusions.

The results in the present paper constitute an interesting warning about the necessity of employing numerical approximation schemes, capable of mimicking the continuous large time dynamical properties of the system, when addressing inverse design and optimal control problems in long time horizons. This was previously observed when dealing with control problems for wave propagation [13,39]. It is interesting to see that the same pathologies persist for the apparently more robust problem of inverse design for inviscid and viscous flows.

This paper is organized as follows. In Section 2 we discuss the existence of minimizers in the continuous setting, distinguishing the viscous and inviscid case. In Section 3 we present the numerical tools that we use for optimization and discretization processes. Then, in Section 4 we solve an optimal control problem in a large period of time for Burgers equation with small viscosity using the gradient descent method and IPOPT. Section 5 revisits the experiments done in Section 4 for the case of inviscid Burgers. In Section 6, we modify some assumptions on the initial setting of the numerical experiments performed in previous sections to test. In Section 7, we visualize functional landscapes and discuss the behavior of the minimizers further. We conclude with some final remarks and perspectives in Section 8.

\section{The COntinuous inVERSE DESIGN PROBlem}

In this section we briefly recall some of the main features of the continuous inverse design or optimal control problem

$$
\begin{aligned}
& \min _{u_{0}} \mathcal{J}_{\nu}\left(u_{0}\right)=\min _{u_{0}} \frac{1}{2} \int_{\mathbb{R}}\left(u^{\nu}(x, T)-u^{*}(x)\right)^{2} \mathrm{~d} x, \\
& \text { subject to } \begin{cases}\partial_{t} u^{\nu}+\partial_{x}\left(\frac{\left(u^{\nu}\right)^{2}}{2}\right)=\nu \partial_{x x} u^{\nu}, & x \in \mathbb{R}, t>0, \\
u^{\nu}(x, 0)=u_{0}(x), & x \in \mathbb{R},\end{cases}
\end{aligned}
$$

distinguishing the viscous, $\nu>0$, and the inviscid case, $\nu=0$.

\subsection{Viscous Burgers}

In the case $\nu>0,(1.1)$ is a parabolic equation that is very well behaved. In particular, given the initial datum in $L^{1}(\mathbb{R}) \cap L^{\infty}(\mathbb{R})$ (actually, $L^{1}(\mathbb{R})$ would suffice), equation (1.1) has a unique solution within the class $C\left([0, \infty) ; L^{1}(\mathbb{R})\right) \cap L^{\infty}(\mathbb{R} \times(0, \infty))$ that is smooth for $t>0$.

The optimization problem (2.1) was addressed in [10] with the aim of adapting the alternating descent method introduced in [9] in the inviscid case. In [10] it was observed that the minimization problem (2.1) has always a solution provided the minimization is performed within a class of a priori bounded initial data, i.e., under an additional constraint of the form

$$
\left\|u_{0}\right\|_{L^{1}(\mathbb{R}) \cap L^{\infty}(\mathbb{R})} \leq K .
$$

Under such a restriction, the proof of the existence of the minimizer follows by the classical Direct Method of the Calculus of Variations.

As far as we know, the existence of the minimizer cannot be guaranteed in the absence of constraints on the initial data, since minimizing sequences could be unbounded. As we shall see, this issue is very closely related to the one-sided Lipschitz condition that the solutions of the viscous Burgers equation satisfy and that imposes a universal threshold within the range of the semigroup at time $t=T$. 
Note however that, due to the parabolic nature of the equation, we can apply classical results of backward uniqueness $[14,31]$ to the linearized model

$$
\begin{cases}\partial_{t} v+\frac{1}{2} \partial_{x}\left(\left(u_{1}+u_{2}\right) v\right)=\nu \partial_{x x} v, & x \in \mathbb{R}, t<T, \\ v(x, T)=0, & x \in \mathbb{R},\end{cases}
$$

$u_{1}$ and $u_{2}$ being two solutions the viscous Burgers equation such that $u_{1}(T)=u_{2}(T)$. Thus, when the target $u^{*}$ is exactly reachable, the initial datum $u_{0}$ leading to it (the minimizer of the functional $\mathcal{J}$ ) is unique, something that fails to be true in the inviscid case [9].

\subsection{Inviscid Burgers}

In the case $\nu=0,(1.1)$ is a nonlinear hyperbolic scalar conservation law. When the initial datum is in $L^{1}(\mathbb{R}) \cap L^{\infty}(\mathbb{R})$ (actually, $L^{1}(\mathbb{R})$ would suffice), equation (1.1) has a unique entropy solution within the class $C\left([0, \infty) ; L^{1}(\mathbb{R})\right) \cap L^{\infty}(\mathbb{R} \times(0, \infty))$. But in the present case, contrarily to the viscous one, smoothness cannot be guaranteed for $t>0$ since solutions may develop shock discontinuities.

In this hyperbolic context, the optimization problem (2.1) was addressed in [9] with the aim of developing specific numerical techniques to treat the cases where optimal solutions develop shock discontinuities. Proceeding as in [9], one can prove that also in the inviscid case the optimal control problem (2.1) has at least a minimizer, provided the initial data are restricted by a constraint of the form (2.2).

Note that one of the main differences between the viscous and the inviscid case is that, for the latter, backward uniqueness does not hold and, consequently, the minimizer is not unique in general.

To conclude this section, we recall the results on the existence of the minimizer, for both viscous and inviscid cases, for the sake of completeness:

Theorem 2.1 (Thm. 2.1 from [9] and Thm. 1 from [10]). Let $\Omega \subset \mathbb{R}$ a bounded interval and $C>0$. Assume that $u^{*} \in L^{2}(\mathbb{R})$ and that

$$
\mathcal{U}_{a d}=\left\{u_{0} \in L^{\infty}(\mathbb{R}), \operatorname{supp}\left(u_{0}\right) \subset \Omega,\left\|u_{0}\right\|_{L^{\infty}(\mathbb{R})} \leq C\right\} .
$$

Then, both the minimization problems

$$
\min _{u_{0} \in \mathcal{U}_{a d}} \mathcal{J}_{0}\left(u_{0}\right)
$$

and

$$
\min _{u_{0} \in \mathcal{U}_{a d}} \mathcal{J}_{\nu}\left(u_{0}\right) \quad(\text { with } \nu>0)
$$

defined in (2.1), have at least one minimizer.

\section{DESCRIPTION OF THE NUMERICAL ALGORITHMS}

In this section, we describe the discrete techniques we employ for solving the optimal control problem (2.1). First, we discuss two numerical discretization methods for the nonlinear PDE, namely the Engquist-Osher (EO) and the modified Lax-Friedrichs (MLF) schemes. We also briefly introduce the discrete version of the inverse design problem.

As we shall see, the wrong long time dynamics introduced by the MLF method or the correct one that EO is capable of mimicking, will be the ultimate reason for the overall performance of the optimization methods, and in particular for the spurious results that one observes in the experiments of Sections 4 and 5 when employing MLF. 


\subsection{Discretization schemes and large-time behavior}

To implement the methods numerically, we opt for a discretization of (1.1) using classical conservative schemes (cf. [22]). As we shall see in the experiments, the choice of the numerical flux is critical when dealing with optimal control problems in large periods of time. Indeed, as pointed out in [26], numerical schemes that add too much viscosity into the hyperbolic system may lead to solutions with the wrong asymptotic behavior. In this section we adapt those results to low-viscosity regimes, in order to understand the underlying phenomena in the previously described optimization problem.

Let us denote spatial nodes $x_{j+1 / 2}=\Delta x(j+1 / 2), j \in \mathbb{Z}$, and time instants $t_{n}=n \Delta t, n \in \mathbb{N} \cup\{0\}$, where $\Delta x, \Delta t>0$ are the mesh size and time-step respectively. We approximate the solution $u$ of (1.1) by a piecewise constant function $u_{\Delta}$ such that

$$
u_{\Delta}(x, t)=u_{j}^{n}, \quad x \in\left[x_{j-1 / 2}, x_{j+1 / 2}\right), t \in\left[t_{n}, t_{n+1}\right),
$$

where

$$
\left\{\begin{aligned}
& u_{j}^{n+1}= u_{j}^{n}-\frac{\Delta t}{\Delta x}\left(g_{j+1 / 2}^{n}-g_{j-1 / 2}^{n}\right) \\
&+\frac{\nu \Delta t}{\Delta x^{2}}\left(u_{j-1}^{n}-2 u_{j}^{n}+u_{j+1}^{n}\right), j \in \mathbb{Z}, n=0, \ldots, N, \\
& u_{j}^{0}=\frac{1}{\Delta x} \int_{x_{j-1 / 2}}^{x_{j+1 / 2}} u_{0}(x) \mathrm{d} x, j \in \mathbb{Z} .
\end{aligned}\right.
$$

Here, $N=\lceil T / \Delta t\rceil$ is the number of time-steps needed to reach $T$. We denote $g_{j+1 / 2}^{n}=g\left(u_{j}^{n}, u_{j+1}^{n}\right)$, where the function $g$ is the numerical flux. In this paper we compare two fluxes:

$$
\begin{aligned}
& \text { Engquist-Osher (EO): } g^{E O}(v, w)=\frac{v(v+|v|)}{4}+\frac{w(w-|w|)}{4} \\
& \text { Modified Lax-Friedrichs (MLF): } g^{M L F}(v, w)=\frac{v^{2}+w^{2}}{4}-\frac{\Delta x}{\Delta t}\left(\frac{w-v}{4}\right) .
\end{aligned}
$$

These schemes are convergent in the classical sense of the numerical analysis (see, for instance, [22]), being of first order of accuracy. Nevertheless, in accordance with the classification in [26], this is not enough when dealing with large-time evolution problems. Given $\Delta x, \Delta t>0$, convergence in the sense of asymptotic dynamics needs to be taken into account too. The choice of the numerical fluxes has been made according to the parabolichyperbolic dichotomy shown there.

Remark 3.1. In the continuous case (see Fig. 1), the changing sign $\mathrm{N}$-waves are the asymptotic profiles as $t \rightarrow \infty$ if $\nu=0$ [32] and intermediate metastable states if $\nu>0$ [29]. In the hyperbolic regime, the key point in the identification of the asymptotic $\mathrm{N}$-wave, which belongs to a two-parameter family, is the preservation of the quantities

$$
p=-\min _{x \in \mathbb{R}} \int_{-\infty}^{x} u^{0}(y) \mathrm{d} y \quad \text { and } \quad q=\max _{x \in \mathbb{R}} \int_{x}^{\infty} u^{0}(y) \mathrm{d} y .
$$

Note that the mass of the initial data $u^{0}$ is, precisely, $M=q-p$. In the parabolic case, there is a period of time when the dynamics are close to the hyperbolic ones. The larger $\min (p, q) / \nu$ is, the longer the diffusion needs to become dominant. Therefore, this needs to be taken into account at the discrete level, since the numerical viscosity can interfere with the correct dynamics of the model.

In our simulations, we have implemented modified Lax-Friedrichs scheme instead of the usual Lax-Friedrichs scheme. It can be shown that Lax-Friedrichs scheme has the maximum amount of numerical viscosity that is allowed by linear stability theory (e.g. [10]) and the scheme becomes unstable in the presence of any physical 


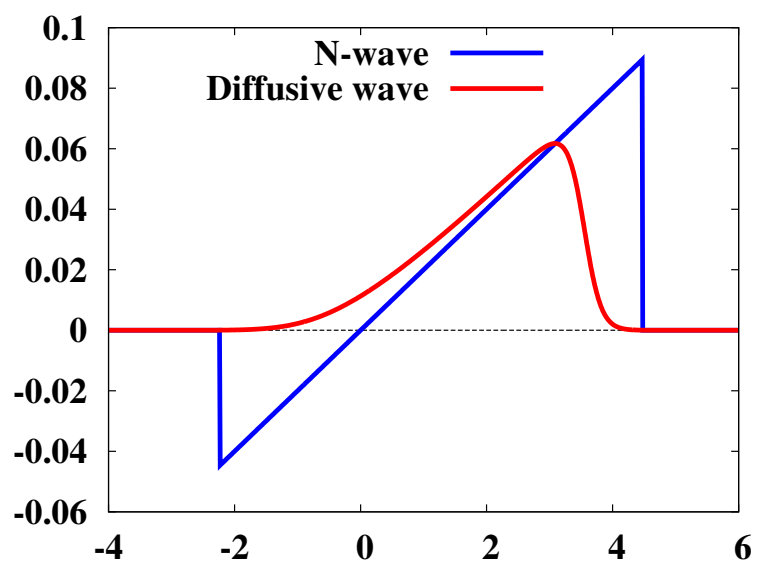

FIgURE 1. Asymptotic profiles for the parabolic $\nu>0$ (red) and hyperbolic $\nu=0$ (blue) cases.

Here we consider $t=50, p=0.05, q=0.2, M=0.15$ and $\nu=0.005$. (Color online).

viscosity $(\nu>0)$. This is not the case for modified Lax-Friedrichs scheme. Nevertheless, both schemes have similar performance in terms of their large-time behavior.

It is well known (e.g. [22]) that (3.1) can be rewritten in its viscous form in the following manner:

$$
\frac{u_{j}^{n+1}-u_{j}^{n}}{\Delta t}+\frac{\left(u_{j+1}^{n}\right)^{2}-\left(u_{j-1}^{n}\right)^{2}}{4 \Delta x}=R\left(u_{j}^{n}, u_{j+1}^{n}\right)-R\left(u_{j-1}^{n}, u_{j}^{n}\right)+\frac{\nu}{\Delta x^{2}}\left(u_{j-1}^{n}-2 u_{j}^{n}+u_{j+1}^{n}\right),
$$

where $R$ is uniquely defined by

$$
R(u, v)=\frac{1}{2 \Delta x}\left(\frac{u^{2}}{2}+\frac{v^{2}}{2}-2 g(u, v)\right) .
$$

In the case of the numerical fluxes that we consider in this paper, we have:

$$
\begin{aligned}
& R^{M L F}(u, v)=\frac{v-u}{4 \Delta t}, \\
& R^{E O}(u, v)=\frac{1}{4 \Delta x}(v|v|-u|u|) .
\end{aligned}
$$

In [26], the authors show that the large-time behavior of $u_{\Delta}$ depends on the degree of homogeneity of the term $R$. In other words, let us assume that there exists $\alpha \in \mathbb{R}$ such that

$$
R(\mu u, \mu v)=\mu^{\alpha} R(u, v), \quad \forall u, v \in \mathbb{R} \text { and } \forall \mu>0 .
$$

It is clear that $\alpha=2$ for Engquist-Osher and $\alpha=1$ for modified Lax-Friedrichs. In the first case, the numerical scheme introduces quadratic numerical viscosity. Thus, as proved in [26], it decays to zero as the $L^{\infty}$-norm of the solution, vanishing in time and preserving the continuous dynamics. On the other hand, the numerical viscosity inherent in MLF is linear and of the order of $\Delta x^{2} / \Delta t$. This means that in the case of $\nu \ll \Delta x^{2} / \Delta t$, the system is driven into a diffusive wave too early and, consequently, continuous metastable states are not reproduced numerically.

Figure 2 shows this pathology of the MLF in the viscous case. It compares the solution to (1.1) with the same initial data at different times, using EO and MLF. Both numerical solutions are similar at early stages. Nevertheless, the one by MLF starts losing the N-wave shape earlier than EO and achieves the diffusive profile in shorter time. 

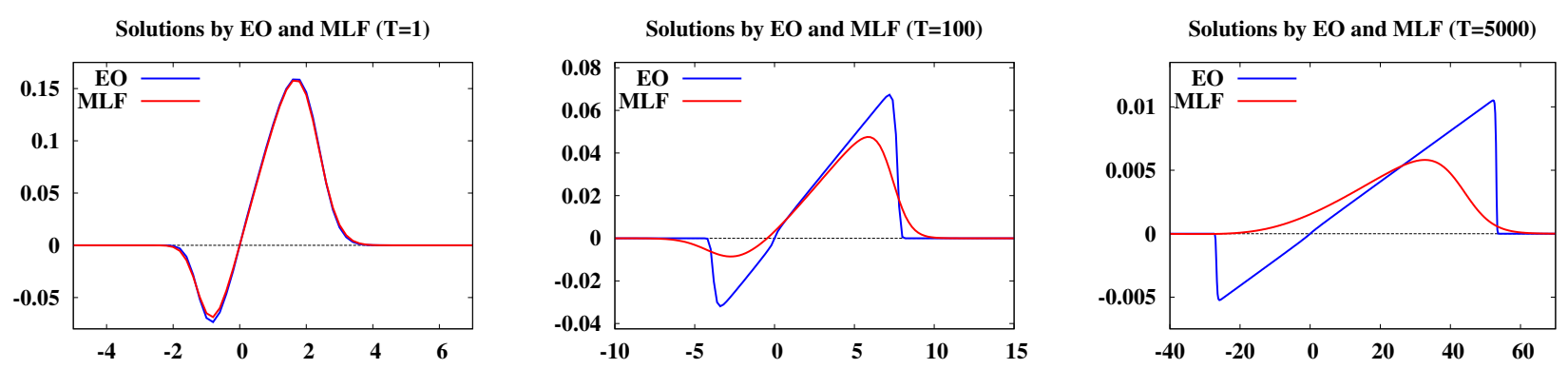

Figure 2. Solutions of (1.1) with $\nu=10^{-6}$ at $t=1, t=100$ and $t=5000$, using (3.1) with $\Delta x=0.2, \Delta t=0.5$ and numerical fluxes EO (blue) and MLF (red). (Color online).

\subsection{The discrete optimization problem}

There are two main ways of addressing optimal inverse design problems like the one we present in this work. On the one hand, in the continuous approach, one first develops the optimization method at the continuous level and then discretizes it. On the other hand, the discrete approach consists in discretizing every element involved in the problem and then optimizing at the discrete level. See, for instance, [18] and the references therein for an introduction on both approaches in the context of design in Computational Fluid Dynamics.

In the simulations done in this work, we follow the discrete approach to optimization. The discretization of (1.1) is done according to (3.1). Regarding the discrete version of (1.2), we consider a simple quadrature rule:

$$
\mathcal{J}_{\Delta}\left(u_{\Delta}^{0}\right)=\frac{\Delta x}{2} \sum_{\mathbb{Z}}\left(u_{j}^{N}-u_{j}^{*}\right)^{2},
$$

where the target function $u^{*}$ has been discretized in the same manner as the initial data $u_{0}$ in (3.1).

The class of admissible data for the discrete minimization problem (3.1)-(3.7) is an approximation of the continuous one, constituted by piecewise constant functions satisfying (2.2). In the inviscid case, no further assumptions are required by virtue of the OSLC satisfied by EO and MLF numerical fluxes [7]. To the best of our knowledge, this condition is no longer available for the viscous case and, thus, an additional restriction is required; namely,

$$
\left\|u_{0}\right\|_{L^{1}(\mathbb{R}) \cap L^{\infty}(\mathbb{R})}+T V\left(u_{0}\right) \leq K .
$$

Within that class of functions, the existence of minimizers for (3.1)-(3.7) is obvious since we are dealing with a finite-dimensional problem. Indeed, if compactly supported initial data are chosen, solutions obtained by explicit schemes have compact support too. The convergence of the discrete minimizers towards the continuous one is less trivial. For the inviscid Burgers equation this fact was already proved in [9], where the authors used the OSLC and a $\Gamma$-convergence argument. Regarding the viscous case, the same procedure is valid, but using BV estimates to work around the lack of OSLC.

Remark 3.2. It is worth emphasizing that in [9] no distinction is made between EO and MLF (or any other numerical scheme, as long as it is monotone and satisfies the OSLC) in terms of their numerical viscosity. In fact, the convergence towards the continuous minimizer is true in both cases. However, as we show in this paper, there is a significant difference in the rate of convergence, at least, in large-time problems.

\subsection{Optimization techniques}

Finally, we present two numerical techniques for solving (3.1)-(3.7). First we recall the classical gradient descent method based on the adjoint methodology. Afterwards, we briefly describe some features of the open source software IPOPT, which uses interior point optimization. 
For the sake of clarity, this section is developed without taking into account restriction (3.8). In fact, this condition is not restrictive in our numerical experiments since the minimizing sequences we obtain do not activate it. We refer to $[11,34]$ for a review about classical methods for constrained problems, such as projected-gradient methods and penalty-function methods.

\subsubsection{Gradient descent by adjoint methodology}

The gradient descent method is a widely spread technique to compute minimizers. This iterative process consists in taking steps in the direction of the gradient of the functional with respect to the controls. Roughly speaking, to minimize a functional $\mathcal{J}$, starting from an initial guess $z_{0}$, one iteratively computes

$$
z_{k+1}=z_{k}-\varepsilon_{k} \nabla \mathcal{J}\left(z_{k}\right), \quad k \in \mathbb{N}
$$

where $\varepsilon_{k}>0$ is the size of the step. Since we are taking the gradient in a negative direction, $\mathcal{J}\left(z_{k+1}\right)<\mathcal{J}\left(z_{k}\right)$ for $\varepsilon_{k}$ small enough, except for the case in which one reaches a minimum in a finite number of iterations: $\nabla \mathcal{J}\left(z_{k}\right)=0$. Observe that, in general, as the minimum is approached, the gradient tends to zero and, hence, the sequence is expected to converge to a (possibly local) minimizer. The convergence of the gradient descent method is well understood when dealing with quadratic coercive functionals and, more generally, for $C^{2}$ functionals exhibiting strict convexity conditions (see [11]). More recently convergence results have been proved for a much larger class of functionals satisfying the so-called Łojaciewicz inequality (see, for instance, [1]).

In our case, the implementation of gradient methods requires a sensitivity analysis of $\mathcal{J}$ with respect to the initial data $u_{0}$. This can be developed in a very classical way for the viscous Burgers equation, because of its parabolic nature and the regularity of solutions for $t>0$. But this is a much more subtle issue in the hyperbolic inviscid frame.

Let us consider perturbations of the form

$$
u_{0}^{\varepsilon}(x)=u_{0}(x)+\varepsilon \delta u_{0}(x),
$$

with $\delta u_{0} \in L^{1}(\mathbb{R}) \cap L^{\infty}(\mathbb{R})$ and $\varepsilon>0$. Then, the solution $u^{\varepsilon}$ of (1.1) with initial data $u_{0}^{\varepsilon}$ is a classical solution too. Moreover, it satisfies

$$
u^{\varepsilon}=u+\varepsilon \delta u+o(\varepsilon),
$$

in a suitable functional setting and, in particular, in $C\left([0, \infty) ; L^{1}(\mathbb{R})\right) \cap L^{\infty}(\mathbb{R} \times(0, \infty))$ and in classes of smooth solutions for $t>0$. Here, of course, $\delta u$ stands for the solution of the linearized system:

$$
\begin{cases}\partial_{t} \delta u+\partial_{x}(u \delta u)=\nu \partial_{x x} \delta u, & x \in \mathbb{R}, t>0, \\ \delta u(x, 0)=\delta u_{0}(x), & x \in \mathbb{R} .\end{cases}
$$

Thus, the Gateaux derivative of $\mathcal{J}$ at $u_{0}$ in the direction $\delta u_{0}$ is

$$
\delta \mathcal{J}\left[\delta u_{0}\right]\left(u_{0}\right)=\int_{\mathbb{R}}\left(u(x, T)-u^{*}(x)\right) \delta u(x, T) \mathrm{d} x .
$$

Now, to simplify this representation of $\delta \mathcal{J}$, we make use of the adjoint methodology. The corresponding adjoint system to (1.1) is

$$
\begin{cases}-\partial_{t} \rho-u \partial_{x} \rho=\nu \partial_{x x} \rho, & x \in \mathbb{R}, t>0, \\ \rho(x, T)=u(x, T)-u^{*}(x), & x \in \mathbb{R}\end{cases}
$$

so that

$$
\delta \mathcal{J}\left[\delta u_{0}\right]\left(u_{0}\right)=\int_{\mathbb{R}} \rho(x, 0) \delta u^{0}(x) \mathrm{d} x .
$$


Therefore, once we have computed the adjoint initial state, this expression provides us an easy way to compute a descending direction for the continuous functional $\mathcal{J}$ :

$$
\delta u_{0}(x)=-\rho(x, 0), \quad x \in \mathbb{R} .
$$

The new perturbed initial data that the gradient descent method yields will be given by

$$
u_{0}^{\varepsilon}(x)=u_{0}(x)-\varepsilon \rho(x, 0) .
$$

The same ideas apply in the inviscid case too, but the issue becomes much more technical because of the lack of regularity of solutions. This leads to the necessity of developing suitable notions of solutions for the linearized systems (see $[5,8,9])$.

Following the discrete approach, we now reproduce the same procedure at the discrete level. The discretization of (2.1) is done according to (3.1)-(3.7). Now, it is easy to obtain the corresponding discrete adjoint system for $(3.1)$ :

$$
\left\{\begin{aligned}
\rho_{j}^{n}= & \rho_{j}^{n+1}+\frac{\Delta t}{\Delta x}\left(\partial_{v} g\left(u_{j}^{n}, u_{j+1}^{n}\right)\left(\rho_{j+1}^{n+1}-\rho_{j}^{n+1}\right)\right. \\
& \left.+\partial_{w} g\left(u_{j-1}^{n}, u_{j}^{n}\right)\left(\rho_{j}^{n+1}-\rho_{j-1}^{n+1}\right)\right) \\
& +\frac{\nu \Delta t}{\Delta x^{2}}\left(\rho_{j-1}^{n+1}-2 \rho_{j}^{n+1}+\rho_{j+1}^{n+1}\right), \quad j \in \mathbb{Z}, n=N-1 \ldots, 0, \\
\rho_{j}^{N} & =u_{j}^{N}-u_{j}^{*}, \quad j \in \mathbb{Z},
\end{aligned}\right.
$$

Remark 3.3. The same analysis as in (3.5) can be done for the discrete adjoint system, reordering (3.12) as follows:

$$
-\frac{\rho_{j}^{n+1}-\rho_{j}^{n}}{\Delta t}-u_{j}^{n} \frac{\rho_{j+1}^{n+1}-\rho_{j-1}^{n+1}}{2 \Delta x}=\bar{R}\left(\rho_{j}^{n+1}, \rho_{j+1}^{n+1}, u_{j}^{n}\right)-\bar{R}\left(\rho_{j-1}^{n+1}, \rho_{j}^{n+1}, u_{j}^{n}\right)+\nu \frac{\rho_{j-1}^{n+1}-2 \rho_{j}^{n+1}+\rho_{j+1}^{n+1}}{\Delta x^{2}},
$$

where

$$
\begin{aligned}
& \bar{R}^{M L F}(\rho, \sigma, u)=\frac{\sigma-\rho}{4 \Delta t}, \\
& \bar{R}^{E O}(\rho, \sigma, u)=\frac{|u|}{2 \Delta x}(\sigma-\rho) .
\end{aligned}
$$

Of course, both systems are linear in the adjoint variables. Note that the numerical viscosity in EO is proportional to the numerical solution $u_{\Delta}$, which decays towards zero as $t \rightarrow \infty$ and, hence, vanishes in time [26]. On the contrary, the amount of numerical viscosity for the MFL adjoint does not depend on the forward solution and, hence, it is kept constant in time, as in (3.5). Thus, the large-time behavior of the discrete adjoint system is affected in the same way as in (3.1).

To minimize (3.7), we will take the descent direction given by:

$$
\delta u_{j}^{0}=-\rho_{j}^{0}, \quad j \in \mathbb{Z} .
$$

This direction is straightforwardly obtained following the same arguments as for the continuous level. Thus, the new initial data $u_{\Delta}^{0, \varepsilon}$ will be given by

$$
u_{j}^{0, \varepsilon}=u_{j}^{0}-\varepsilon \rho_{j}^{0}, \quad j \in \mathbb{Z}
$$

for some $\varepsilon>0$ small enough. We refer to Section 6 for a discussion on the choice of the step-size $\varepsilon$.

Solving the adjoint system (3.12) requires knowing the solution of (3.1) at every time instant. For large-time problems, storing the time-history solution can be computationally prohibitive. In our experiments we did not 
need to use such strategies, but problems like the sonic-boom minimization could require additional efforts. For a detailed implementation of the adjoint methodology including memory-saving techniques, such as backtracking, see $[21]$.

Moreover, one cannot always expect this iterative process to finish successfully (i.e., finding an exact minimizer) in a reasonable time. When this occurs, additional stopping criteria need to be set. For instance, one could stop the iterations by specifying:

- The smallest difference between two consecutive approximations of the optimal solution.

- The smallest value that the norm of the gradient can take.

- The smallest value that $\varepsilon$ can take without descending.

- The absolute error between the approximation and the optimal solution, if this is known a priori.

Note that all of them are related to the proximity to a minimizer, either local or global. In our experiments we opt for the third one, due to its algorithmic simplicity. We refer to [34] for an extended summary about these and other method-specific stopping criteria. In conclusion, each iteration for the gradient descent method is as shown in Algorithm 1.

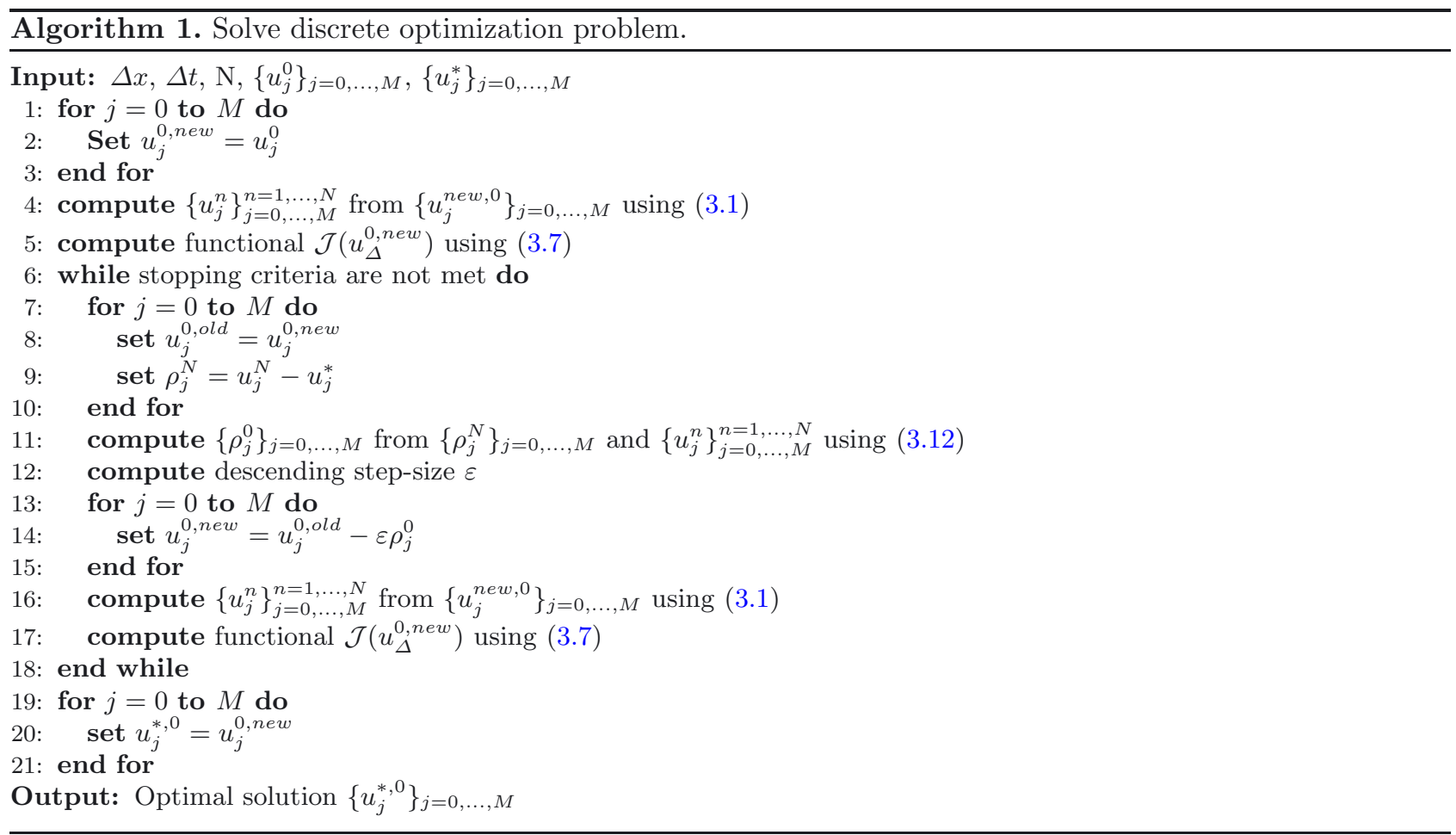

\subsubsection{The interior-point optimization method with IPOPT}

IPOPT has been developed for efficient optimization of large-scale nonlinear programing. It implements interior-point or barrier methods, which provide a more attractive alternative to active set strategies in optimizing problems with a large number of inequality constraints. IPOPT can converge to an optimal solution even from infeasible starting points through using a filter line-search method. The underlying concept of filter methods is to accept trial points along the line-search if they improve the objective function or improve the constraint violation instead of a combination of these two criteria defined by a combined merit function. The filter 
concept excludes the possibility of the solution to alternate between two iteration points, so that one improves the objective function while the other avoids constraint violations (see [37]).

The optimal control problem discussed in (2.1) is formulated as a minimization problem with constraint equations in the form of Partial Differential Equations (PDE). The PDE constraint is reduced to a set of nonlinear algebraic equations through (3.1). The resulting nonlinear optimization problem is solved using a primal-dual interior point algorithm implemented in the open-source IPOPT code.

Setting up a problem in IPOPT, as a general purpose optimization package, usually requires less effort and time in comparison with developing an adjoint solver for a specific equation. However, IPOPT runs are more computationally intensive due to the fact that IPOPT assembles and solves a linear system for the solution of Karush-Kuhn-Tucker (KKT) optimality condition in each iteration if compared with less intensive backward solution of the adjoint system adopted in GDM. IPOPT needs information on functional gradient, Jacobian of constraints, and Hessian of augmented functional to form and solve the optimality condition using a Newton method. In our implementation, we used automatic differentiation capabilities of AMPL to compute all required derivatives [15].

Regarding the stopping criterion, the one in IPOPT is based on the norm of the residual in the optimality condition. At the optimum point, the optimality condition has no residual. Optimization algorithm in IPOPT stops when the norm of the residuals in the optimality condition gets smaller than a predefined tolerance, the default value being $10^{-8}$.

\section{Numerical experiments for the Viscous Burgers Equation}

In this section we present a first numerical experiment showing the influence of the numerical viscosity outlined in the previous section. The main aim is to emphasize that ignoring the dynamics of the continuous model at the numerical level can produce undesired results in optimal control problems in large time horizons.

On one hand, we show that the gradient descent method performs successfully whenever the numerical flux and the mesh sizes are chosen appropriately. On the other hand, we present examples where excessive numerical viscosity dominates the physical one. In those cases, we show that IPOPT produces solutions with amplified spurious numerical oscillations. We refer the reader to Section 4.4 for a comparison between the different approaches.

\subsection{Description of the numerical scheme}

In order to show the numerical phenomena described above, let us choose the following target function:

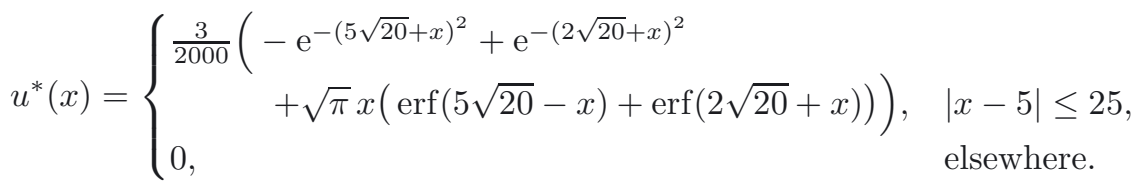

The final time $T$, and viscosity $\nu$ have been chosen to be self-similar with the sonic-boom propagation in the atmosphere which is characterized by low value of viscosity present in a long time duration. In doing so, we selected the dimensionless number of $\frac{T \nu}{\rho|u|^{2}}$ where $\rho$ represents density. $\rho=1$ in Burgers equation in (1.1). In other words, we chose $T=50, \nu=10^{-4}$ such that the dimensionless number is of the same order of magnitude as that of the sonic boom propagation in the atmosphere.

Regarding the discretization parameters, in each experiment we first fix the mesh-size $\Delta x$. Then, the time-step is chosen according to the stability condition of the scheme, that is

$$
\frac{\Delta t}{\Delta x} \max _{j \in \mathbb{Z}}\left|u_{j}^{0}\right|+2 \nu \frac{\Delta t}{\Delta x^{2}} \leq C_{s t a} .
$$


Target function

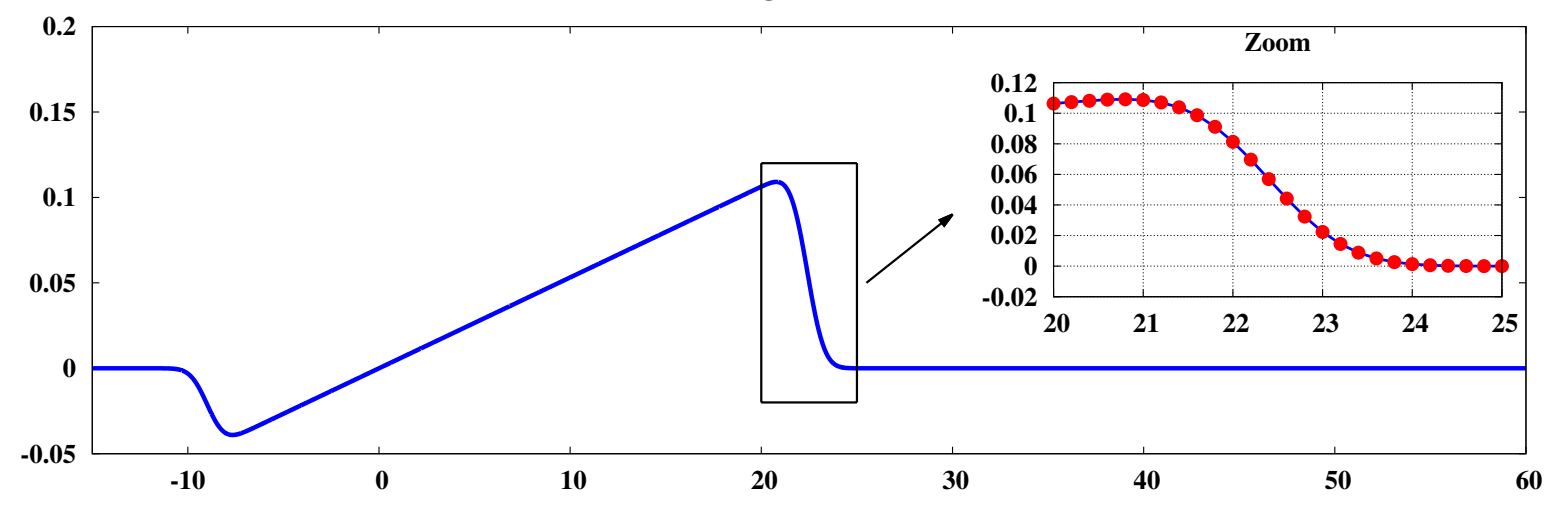

Figure 3. Target function and sampling for $\Delta x=0.2$.

In the case of EO, $C_{s t a}=1$, while for MLF, $C_{s t a}=1 / 2$ (e.g. $\left.[10,22]\right)$. However, in our experiments we use $C_{s t a}=1 / 2$ in both cases, unless otherwise specified, which allows us to better compare their performances. The computational domain is chosen large enough to avoid reflection from boundaries.

Remark 4.1. Note that this target, being of compact support, is not exactly reachable for the viscous Burgers equation. Indeed, in view of the Hopf-Cole transformation ([38], Chap. 4), it can be seen that the value of solutions at the final time cannot vanish on an open set. Observe also that this target is not too steep (see Fig. 3). In that way, we avoid the numerical consequences on the behavior of the algorithms caused by quasi-shocks [10] and there is no need to increase the number of nodes across them [19]. This will allow us to better focus on the impact of the large-time behavior on the performance of optimization techniques.

\subsection{Results using GDM}

We initialize the gradient descent method from the function $u_{0}=0$. For each of the numerical fluxes, we repeat the experiment using different mesh sizes, namely $\Delta x=0.1,0.2,0.4,0.8$.

As we observe in Figure 4, with EO we are able to compute a quite satisfactory minimizer even for $\Delta x=0.8$, which returns an optimal value below $10^{-5}$. Making $\Delta x$ smaller, the obtained result is even better (blue lines in Fig. 6) but yielding always a similar minimizer. This shows the robustness of the method, the optimal solution being similar in the four cases.

Nevertheless, as we expected, the GDM does not perform so well when coupled with MLF. In Figure 5 we observe that large enough mesh-sizes introduce small over-shootings around the most steep regions of the target function. This is due to the numerical viscosity that MLF introduces, which is proportional to $\Delta x^{2} / \Delta t$. When that ratio is comparable with $\nu$, the dynamics of the numerical solution cannot preserve the positive and negative masses (3.4) along the control horizon $[0, T]$. As a consequence, the obtained initial datum requires those oscillations in order to maintain the $\mathrm{N}$-wave shape after large periods of time.

Figure 6 shows that, furthermore, the results obtained this way are always worse than the ones obtained using EO. Note that, despite the oscillations, the optimal solution approaches the one obtained using EO as $\Delta x$ gets smaller. This is in agreement with the results of Section 3 on the convergence of discrete minimizers towards the continuous ones. But, as expected, convergence is much slower as the mesh-size decreases for the MLF.

Nevertheless, the values corresponding to MLF stay always above the ones obtained using EO after the same number of iterations. In fact, GDM+MLF meets the stopping criteria much before than GDM+EO. 

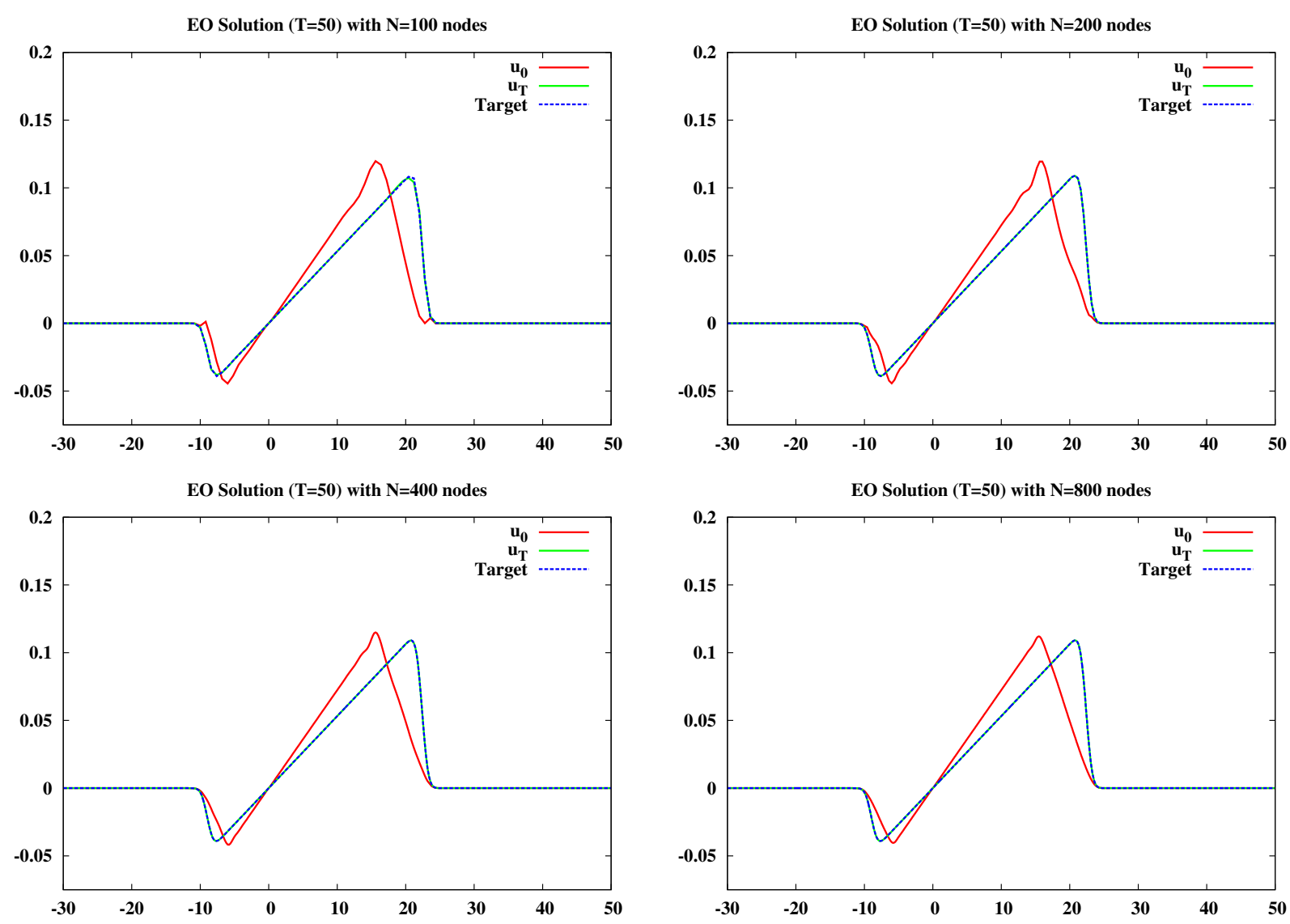

Figure 4. Optimal solutions (red) for (2.1) with $\nu=10^{-4}$ and their corresponding state at time $T=50$ (green) compared to the target (blue), using GDM+EO. From left to right and top to bottom, $\Delta x=0.8,0.4,0.2$ and 0.1 respectively. (Color online).

\subsection{Results using IPOPT}

In this section, the numerical experiment for viscous Burgers is solved with IPOPT using both EO and MLF numerical fluxes introduced in equations (3.2) and (3.3). As explained in Section 3, the algebraic constraints consists of the discretized form of the Burgers equation shown in equation (3.1).

The optimization results obtained with IPOPT are shown in Figure 7 for the EO discretization and in Figure 8 for the MLF scheme. The initial data $u^{0}$ that is sought is shown in red solid lines, while the final solution at time $T$ and the target function $u^{*}$ are plotted respectively in green and blue lines, being indistinguishable within plotting tolerance. For each scheme, the optimization problem is solved for four different cell sizes from coarse to refined meshes corresponding to $\Delta x=0.2,0.1 \overline{3}, 0.10,0.08$. Both EO and MLF fluxes achieve a discrete initial condition which reduce the functional to values less than $10^{-8}$.

The spurious oscillations in initial data obtained with MLF are due to the numerical viscosity that MLF scheme introduces. In cases where numerical viscosity in MLF is comparable with the physical viscosity, MLF discretization can alter the dynamics of the continuous system, especially in long time horizons. This fact constitutes a warning regarding the selection of cell size and time-step when MLF discretization is employed. 

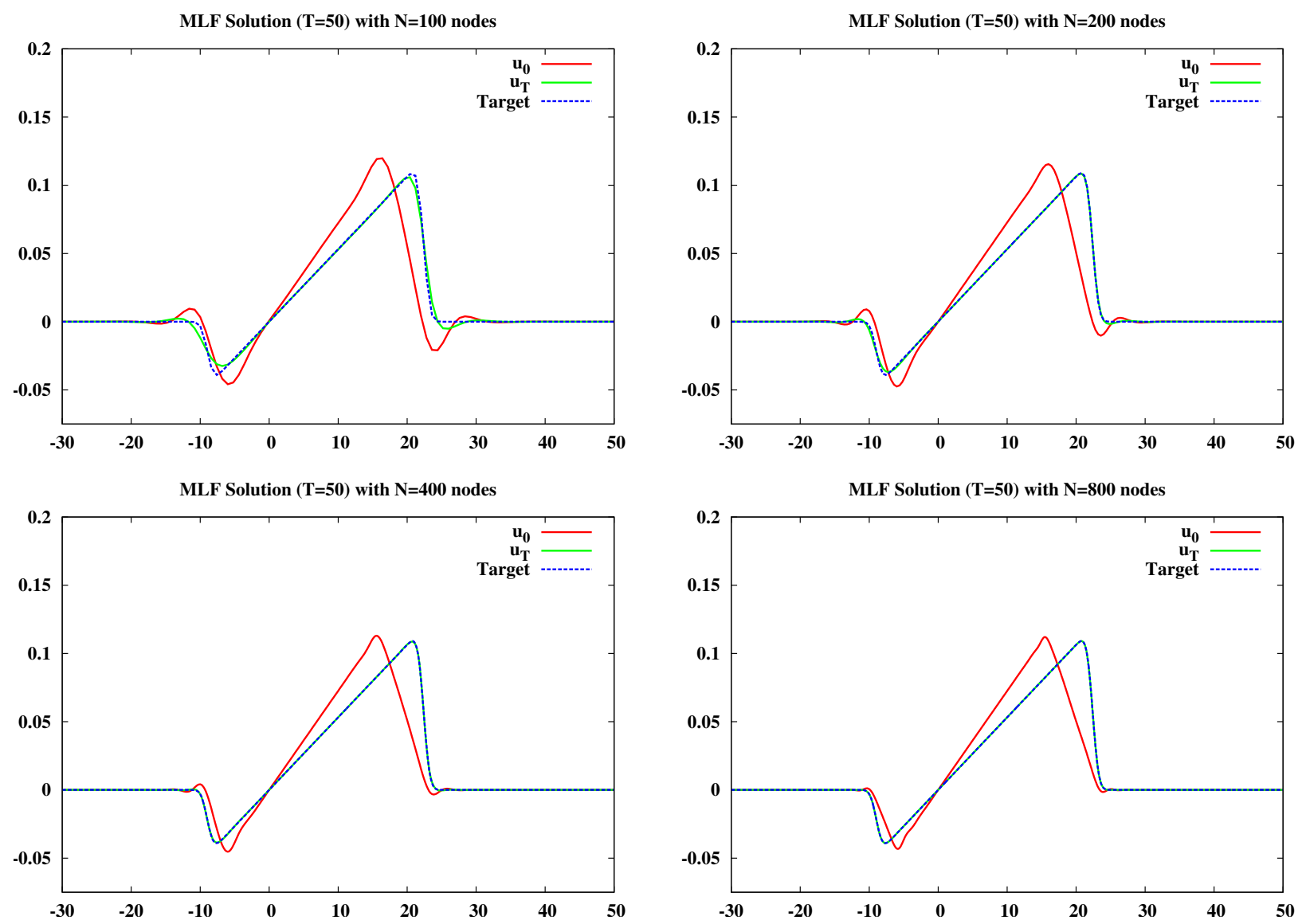

FIGURE 5. Optimal solutions (red) for (2.1) with $\nu=10^{-4}$ and their corresponding state at time $T=50$ (green) compared to the target (blue), using GDM+MLF. From left to right and top to bottom, $\Delta x=0.8,0.4,0.2$ and 0.1 respectively. (Color online).
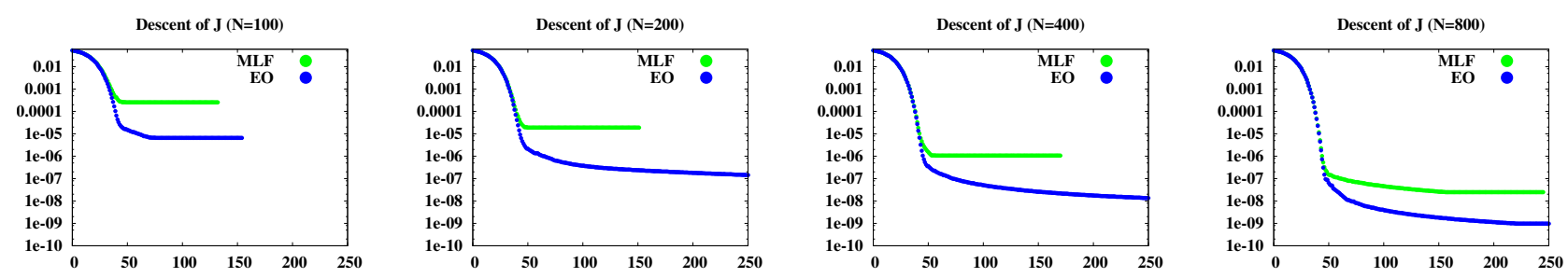

Figure 6. Descent of the functional $J$ using GDM coupled with EO and MLF. We take, respectively, $\Delta x=0.8, \Delta x=0.4, \Delta x=0.2$ and $\Delta x=0.1$ for the case $\nu=10^{-4}$ and $T=50$. 

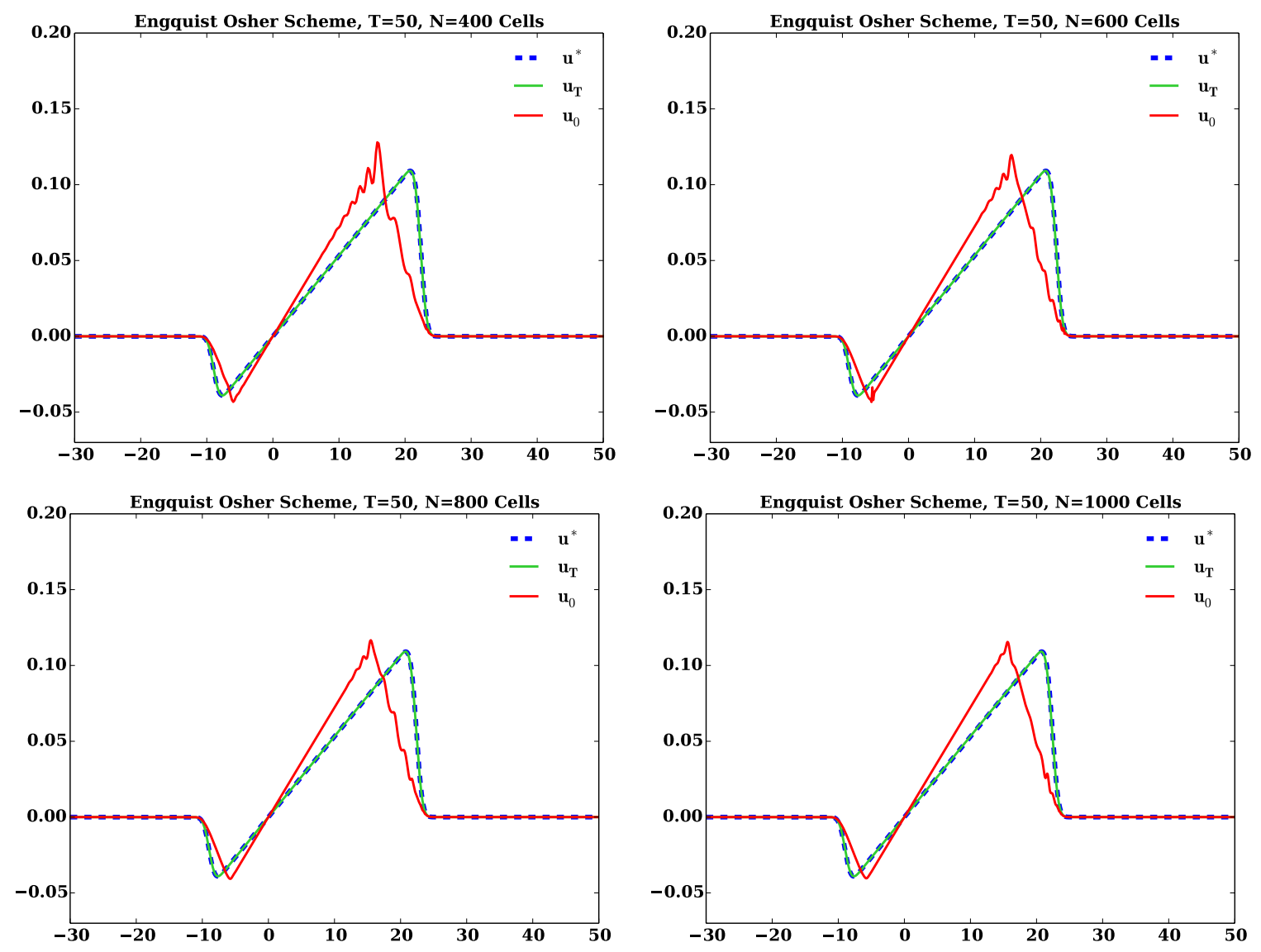

Figure 7. Optimal solutions for (2.1) with $\nu=10^{-4}$ and $T=50$, using EO flux discretization. From left to right and top to bottom, $\Delta x=0.2,0.1 \overline{3}, 0.10,0.08$ mesh sizes respectively.

Indeed, from the inverse problem point of view, extra numerical viscosity in MLF makes the inverse problem even more ill-posed.

Remark 4.2. To show that oscillations in MLF results are artifacts of the numerical viscosity, the MLF initial data are evolved with a Burgers equation solver which adopts EO discretization and the result is shown in Figure 9 on the left. The fact that EO retains oscillations from MLF initial condition corroborates the oscillations in MLF results do not have any physical significance with regard to the Burgers equation and they are merely undesirable artifacts of numerical viscosity of MLF. On the right plot of Figure 9, the optimization results obtained from EO are evolved with MLF. As observed, the numerical viscosity in MLF solver causes more dissipation in final solution $u$ when it is compared with the target function $u^{*}$. In summary, the optimal initial datum obtained by one method is not necessarily the best fit for the functional if it is evolved with a scheme that introduces a different degree of numerical viscosity. Similar issues were already detected in [17], regarding the different amount of numerical viscosity when solving the forward and the adjoint systems, respectively.

\subsection{Comparing GDM and IPOPT results}

Here, we compare qualitatively the optimization results of GDM and IPOPT. The results obtained from GDM and IPOPT are quite similar in the case of EO. However, in the case of MLF, results obtained from 

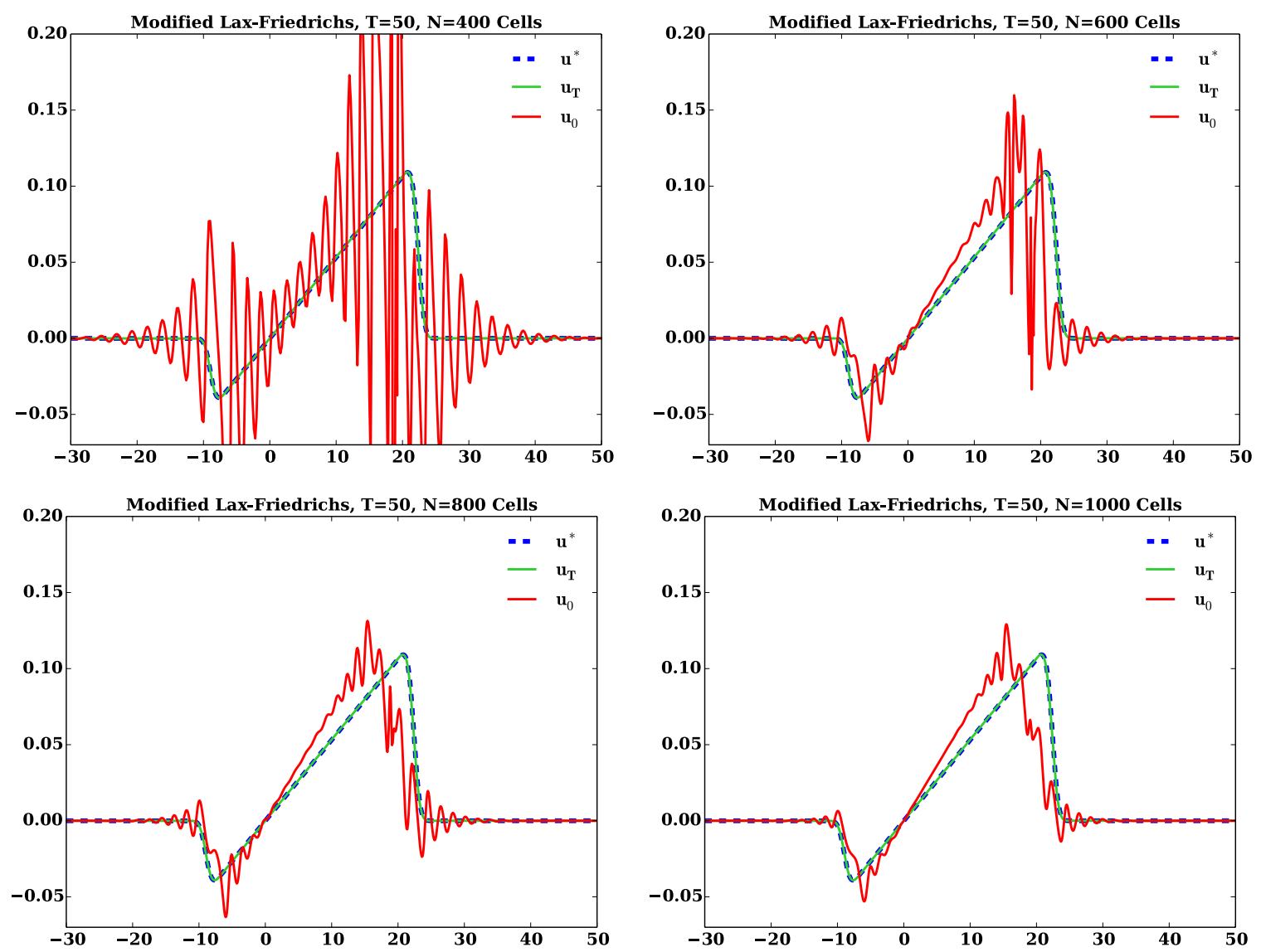

FiguRE 8. Optimal solutions for (2.1) with $\nu=10^{-4}$ and $T=50$, using MLF flux discretization. From left to right and top to bottom, $\Delta x=0.2,0.1 \overline{3}, 0.10,0.08$ mesh sizes respectively.

IPOPT contain more oscillations when compared with those of GDM. It is seen that even for the smallest cell size corresponding to $\Delta x=0.08$, IPOPT still maintains oscillations. From the frequency domain perspective, as cells are refined, IPOPT will have the opportunity to include high frequency oscillations that were not resolved on coarser meshes. IPOPT via using a higher order optimization scheme is capable of including high oscillations.

In the case of GDM, which employs gradient information calculated by solving the adjoint equation, it does not allow high frequency oscillations due to the boundedness of backward adjoint solutions as we iterate in time. This observation is consistent with the ill-posedness of the backward solution for viscous processes. Lower order convergence of gradient method with adjoint methodology acts as a regularizing effect for the ill-posed problem of the numerical viscosity term introduced by MLF method.

Another interesting observation regarding the extent of oscillations observed in GDM methods will be discussed in Section 6 in relation with the choice of the step-size in descent methods. It will be shown that adopting big step-size $\varepsilon$ in the line-search method may result in oscillatory results even in GDM+EO case as shown in Figure 15. The aggressive optimal step-size may not guarantee a smooth descent toward the solution in the case of Burgers equation. The sensitivity of the optimization algorithm to the initialization and to the step-size have been further elaborated in Section 6. 

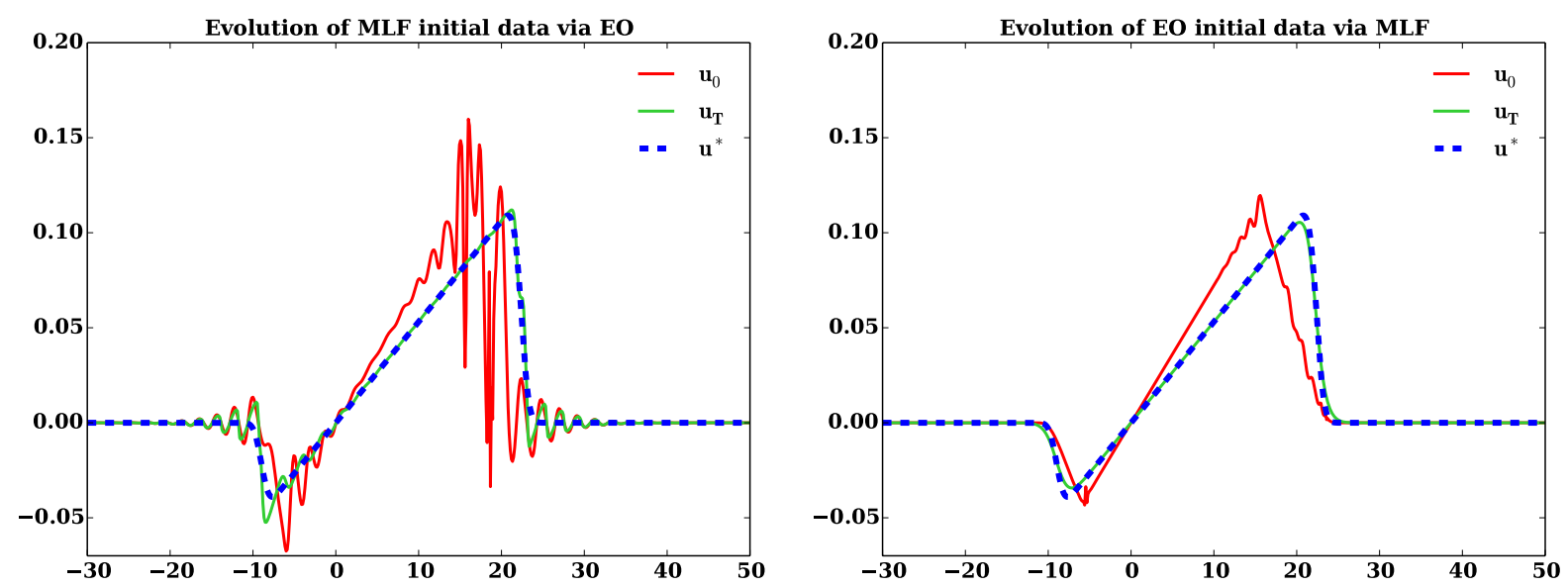

Figure 9. Optimal solutions (red lines) for (2.1) with $\nu=10^{-4}$ and $T=50$ obtained from IPOPT +MLF (left) and IPOPT+EO (right), using $\Delta x=2 / 15$. Initial data obtained with MLF is evolved with EO discretization and vice versa, reaching solutions (green lines) which deviate from the target function (blue lines). The optimal initial data obtained for one scheme is not necessarily the best fit for the functional if it is evolved in time with a different scheme. (Color online).

\section{NUMERICAL EXPERIMENT FOR INVISCID BURGERS EQUATION}

So far, we have focused on the viscous Burgers equation with small viscosity. In this section, we revisit the experiment shown in Section 4, but for the inviscid case, in which $\nu=0$.

It is important to recall that the inviscid Burgers equation is not time reversible. Thus, in principle, not all states lead to a unique minimizer. Let us remark also that, in the absence of viscosity, shock discontinuities may arise in finite time even for smooth initial data. Nevertheless, the target function (4.1) has been chosen in order to avoid the severe influence of shocks on the optimization process to the extent possible. We refer to discussion in Section 6 for more details about this issue in the context of the small viscosity and quasi-shocks, which can be extended to the inviscid case.

The results obtained from the gradient descent method, using both EO and MLF, are shown in Figure 10. In Figure 11 we display the results for the same experiment using IPOPT. One can immediately notice that neglecting the physical viscosity does not cause any substantial difference in the performance of the algorithms. As in the viscous case, EO performs well, while spurious oscillations pollute MLF results.

Remark 5.1. The conclusions of this experiment and the ones of the following section can also be extended to the targets with shocks. Note that in those cases, the large-time effects may remain hidden due to the presence of shocks. In Figure 12 one can observe EO performing better than MLF even when the target function is discontinuous.

\section{Other VARIANTS OF NUMERICAL EXPERIMENTS}

It is well known that iterative algorithms can be sensitive to the underlying assumptions considered in their initialization. This section constitutes a warning on how results could be affected if intrinsic characteristics of the optimal control problem (mainly, those related to the PDE and its discretized version) are not taken into account. We revisit the experiment of Section 4, based on the viscous case, while modifying the initial settings of the numerical optimization process. All the same, the conclusions can be extended to the inviscid case too. In particular, we consider the following variants:

- Sensitivity to the initialization of optimization algorithms.

- Choice of the step-size in descent methods. 

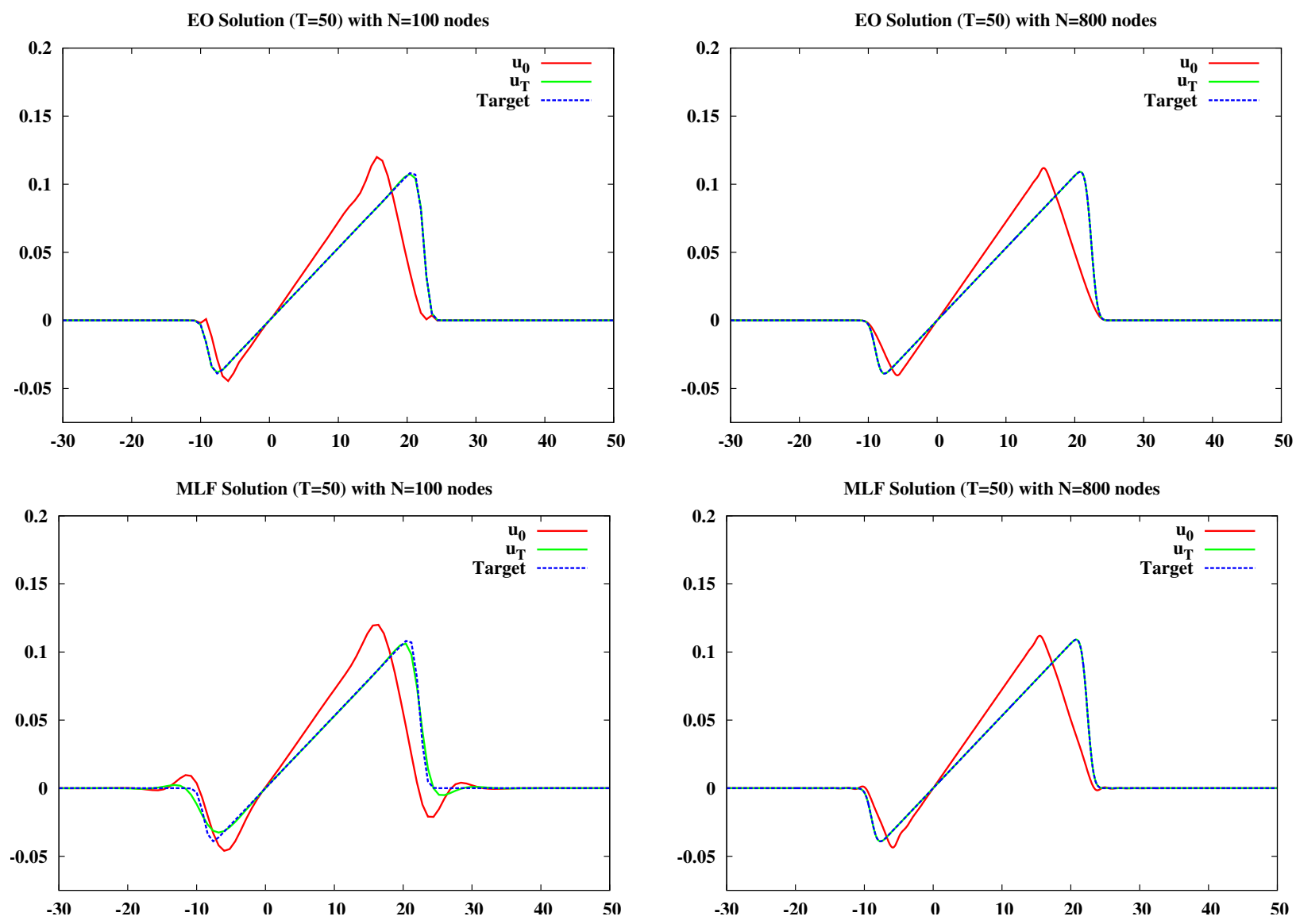

Figure 10. Initial data $u^{0}(x)$ for inviscid Burgers equation obtained from GDM+EO (top) and GDM+MLF (bottom). We use $\Delta x=0.8$ (left) and $\Delta x=0.1$ (right).

- Reducing the time-step.

- Prescribing an unreachable target function.

\subsection{Sensitivity to the initialization of the optimization algorithm}

In all numerical experiments presented so far, the initial data considered to initialize the optimization algorithm were taken to be identically zero. To evaluate the impact of this choice, we solve (3.1)-(3.7) for target (4.1) starting from other initial guesses: a sinusoidal function and a step function. The results obtained with GDM are shown in Figure 13, while the ones for IPOPT are shown in Figure 14.

It is worth underlying the numerical results obtained by means of the step function initialization. This choice of the initialization gives rise to a spike on the optimal initial datum regardless the method we employ. As a matter of fact, the presence of discontinuities in the initial data, even in the viscous Burgers equation, is a delicate issue to deal with (e.g, see [10]). Once they are raised, they have the tendency to persist when employing discrete approaches as in here.

In terms of the value of the functional $\mathcal{J}$, the presence of this kind of spikes in the initial data does not bear any consequences, since it is attenuated during the evolution process. Let us remark that, in what concerns the 

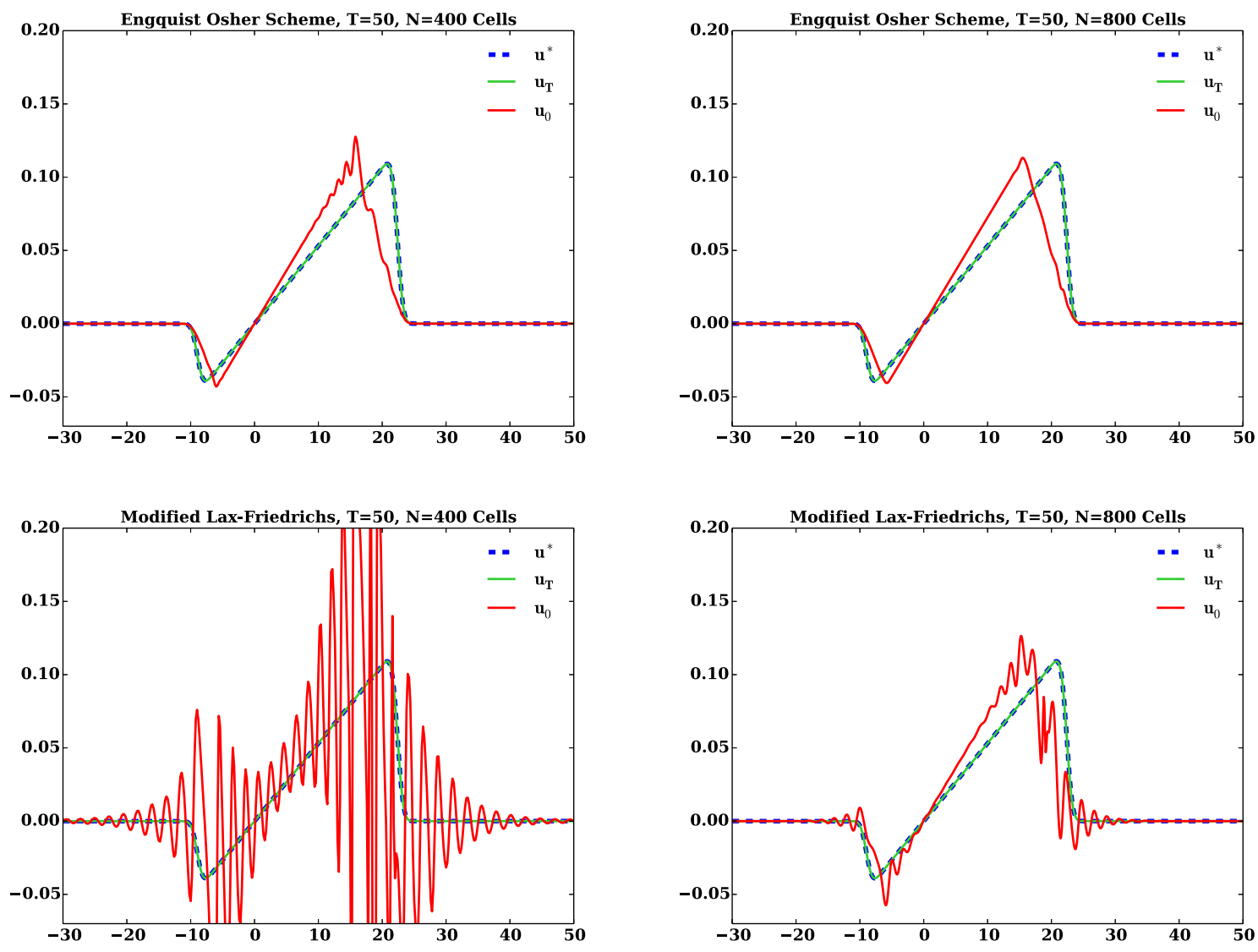

FIgURE 11. Initial data $u^{0}(x)$ for inviscid Burgers equation obtained from IPOPT to fit target function $u^{*}(x)$ at time $T=50$ using EO (top) and MLF (bottom) flux discretizations. We use $\Delta x=0.2($ left $)$ and $\Delta x=0.1$ (right).

EO Solution $(\mathrm{T}=50)$ with $\mathrm{N}=400$ nodes

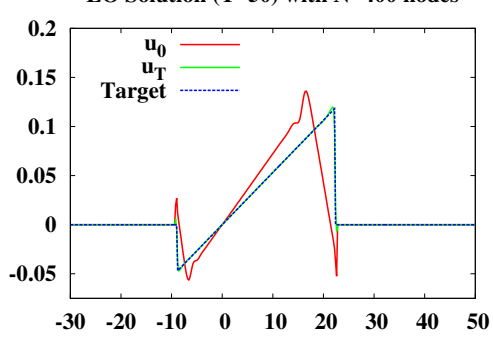

LFM Solution ( $\mathrm{T}=\mathbf{5 0})$ with $\mathrm{N}=400$ nodes

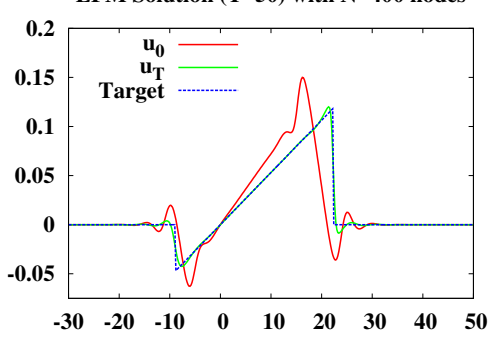

Descent of $\mathrm{J}(\mathrm{N}=\mathbf{4 0 0})$

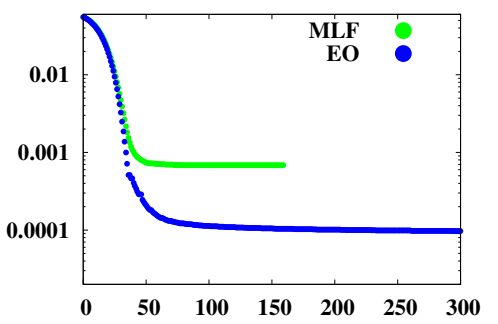

Figure 12. Obtained initial data for a discontinuous target function under inviscid Burgers dynamics, using EO (left) and MLF (middle) with $\Delta x=0.2$. Resulting from the presence of shocks, overshootings appear in their neighborhood, mixing large-time effects and oscillations due to discontinuities. In any case, the functional clearly diminishes more in the case of EO. 

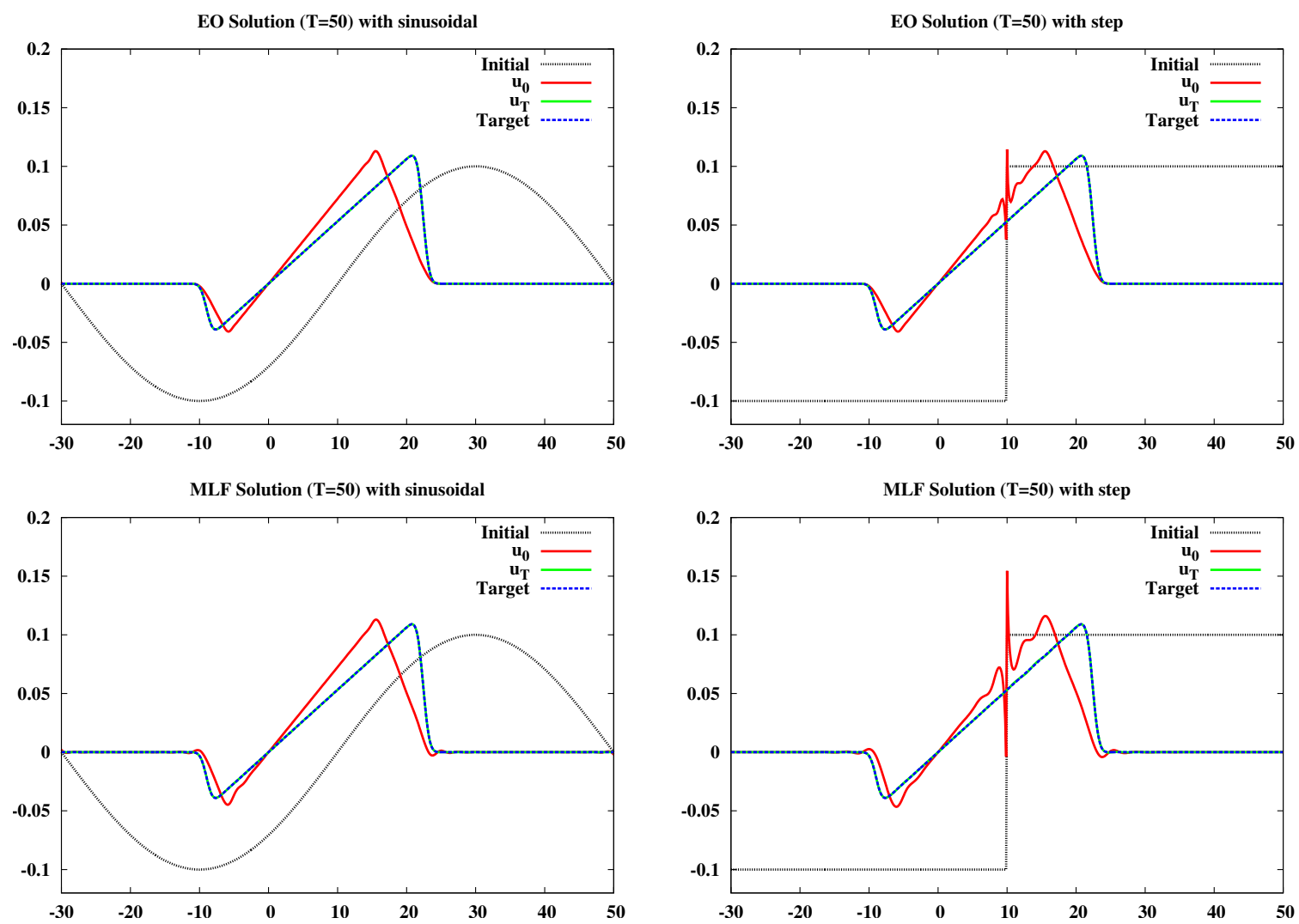

FiguRE 13. Optimal initial data $u^{0}(x)$ obtained with the GDM, using sinusoidal (left column) and step function initialization (right). We compare EO (top) and MLF (bottom) numerical fluxes. The experiment was performed for $\Delta x=2 / 15, T=50$ and $\nu=10^{-4}$.

inviscid dynamics, $p$ and $q$ in (3.4) are not very sensitive to this kind of perturbations; a conclusion that can be extrapolated to the viscous case. Nevertheless, misplaced discontinuities and, more generally, quasi-shocks affect severely the optimization process.

\subsection{Choice of the step-size in descent methods}

Gradient descent methods based on the adjoint equation, that we introduced in Section 3, have several variations depending on the way of choosing the descending direction or the step-size. We refer to $[11,34]$ for basic reference about some of those. The main objective of these variations is, usually, to obtain a better convergence rate. In this section we want to highlight that a quick descent at the very first iterations does not imply a better global convergence. In other words, being conservative at the beginning can lead into a better global performance of the optimization process.

In the method that we introduced in Section 3 we did not specify the way one should choose $\varepsilon$ in (3.14). Taking $\delta u^{0}=-\rho(0)$ and a sufficiently small $\varepsilon$ guarantees a descending step, even if it might not be optimal. In the experiments of this paper concerning the GDM, in each iteration, we have chosen $\varepsilon$ in the following manner:

$$
\varepsilon_{\text {new }}=0.5^{r}\left(1.2 \varepsilon_{\text {old }}\right)
$$



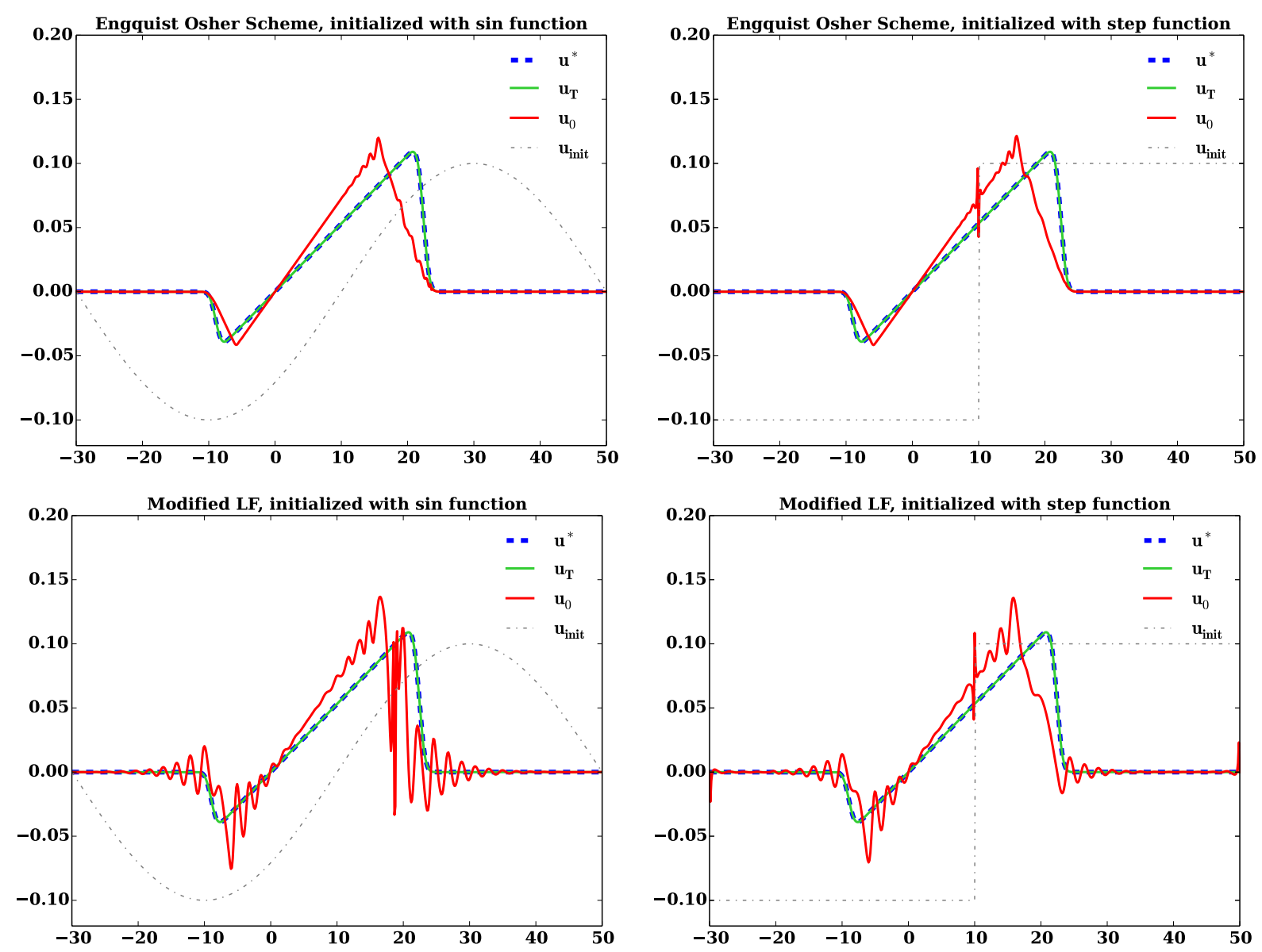

FiguRE 14. Optimal initial data $u^{0}(x)$ obtained from IPOPT to fit the target function $u^{*}(x)$, using sinusoidal (left column) and step function initialization (right). We compare EO (top) and MLF (bottom) numerical fluxes. The experiment was performed for $\Delta x=2 / 15, T=50$ and $\nu=10^{-4}$.

with the smallest $r=0,1,2, \ldots$ such that

$$
\mathcal{J}\left(u_{\Delta}^{0, \text { new }}\right) \leq \mathcal{J}\left(u_{\Delta}^{0, \text { old }}\right)
$$

Remark 6.1. The chosen step-size is enough to show the pathological results that numerical viscosity produces in long-time horizon problems. Note that we do not claim this step-size to be the best choice for this type of problems. Indeed, steepest descent or conjugate gradient methods, together with an adequate line-search strategy, should be more efficient generally. However, even in those cases, the same issues described along this paper would arise too.

A bigger value for the initial $\varepsilon_{0}$ will imply a deeper modification of the initial data in the earlier steps and, possibly, a bigger decrease of the functional. Nevertheless, this does not guarantee a better convergence. As a matter of fact, in Figure 15 we present the initial data obtained in the experiment of Section 4 after the first iterations of the GDM+EO method with $\Delta x=0.1$. In this case, we have chosen the step size according to (6.1), 

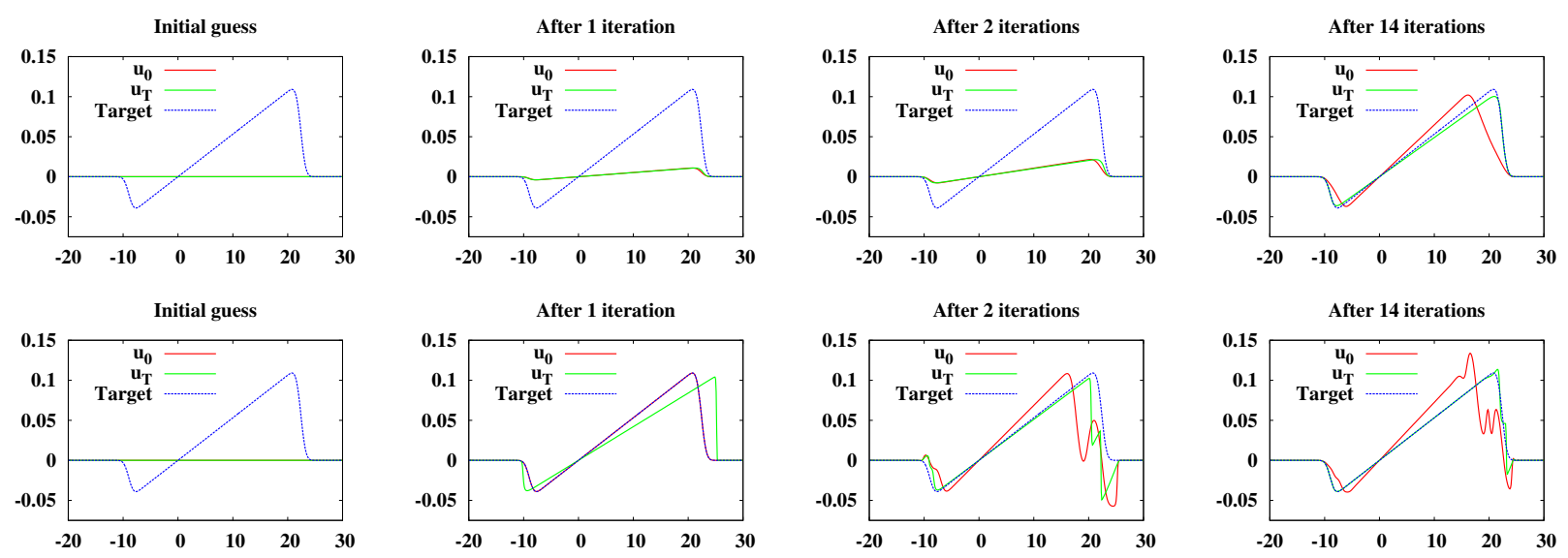

FIGURE 15. Initial data obtained after 1, 2 and 14 iterations of the GDM+EO method, started from $u_{\Delta}^{0}=0$, using $\varepsilon_{0}=0.1$ (top) and $\varepsilon_{0}=1$ (bottom) as initial step-sizes for (6.1). As shown in Figure 16, the descent gained in the first iteration using a bigger step is lost due to the creation of a misplaced quasi-shock.
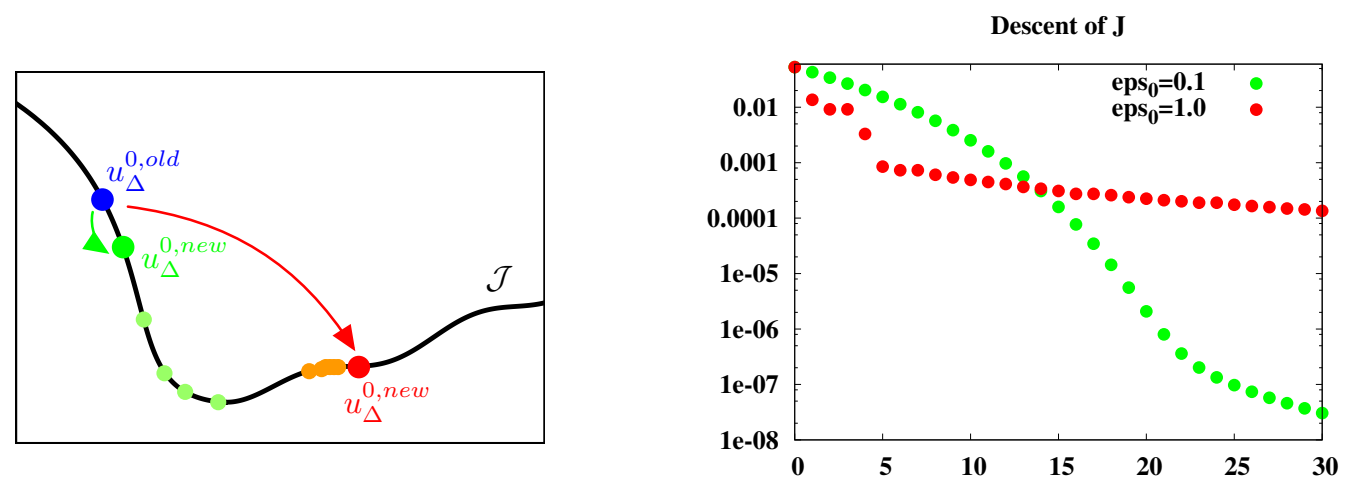

Figure 16. A big step at the beginning can take the descending path through flat regions. For instance, the creation of a quasi-shock in an incorrect place, as in Figure 15, can lead to a slower convergence. On the right, evolution of the functional using GDM+EO with $\varepsilon_{0}=0.1$ (green) and $\varepsilon_{0}=1$ (red) as initial step-sizes. (Color online).

but starting from $\varepsilon_{0}=0.1$ and $\varepsilon_{0}=1$. One can clearly notice that, after the first iteration, the initial data obtained using $\varepsilon_{0}=1$ produces a closer solution to the target function.

The cost of creating a misplaced quasi-shock is high and it is reflected in the subsequent iterations. Dealing with quasi-shocks in optimal control problems like the one we present in this paper is a very hard task. Even if solutions of (1.1) are continuous for $t>0$, very steep regions (quasi-shocks) may arise if $\nu$ is small. From the numerical point of view, these behave almost like shocks and, thus, their presence in incorrect places is not desirable. Since the gradient descent method of Section 3 is designed from the point of view of smoothness of solutions, it finds difficult to bypass quasi-shock misplacements. In a figurative manner, this can be represented as in Figure 16. Note that taking a big step can reduce drastically the functional, but in exchange for a badly located quasi-shock. This ends up driving the descending path through flatter regions that are harder to overcome. 
Remark 6.2. Let us emphasize that this issue becomes critical in the presence of shocks or quasi-shocks. The adjoint methodology seems to recover a rarefaction wave from which the wavefront arises in the end. Thus, big perturbations of the initial data in the first steps end up with oscillations like the ones shown in Figure 15, deeply affecting the efficiency of the algorithm. Of course, this issue could be mitigated if more complex techniques, capable of handling shocks, were used. For instance, the alternating descent method developed for the hyperbolic case in [9] and applied to the parabolic case in [10], allows to move the location of the discontinuities in the initial data, reducing the number of iterations drastically. But the extension to solutions without discontinuities is an interesting application that remains open.

\subsection{Reducing the time-step}

So far, we have chosen the largest time-step $\Delta t$ that (4.2) allows, reducing $\Delta x$ to illustrate the convergence of the algorithms. However, one could think on taking a smaller time-step instead, choosing $\Delta t$ such that

$$
\Delta t<\Delta t_{\max }=C_{\text {sta }} \frac{\Delta x^{2}}{\Delta x \max _{j \in \mathbb{Z}}\left|u_{j}^{0}\right|+2 \nu} .
$$

In terms of stability of the numerical schemes, this is permitted. But, as we show below in Figure 17, the optimization results can be severely affected by this fact.

In the case of EO, a smaller time-step does not have much influence on the results. On the contrary, MLF is strongly affected by this situation. Let us recall that the large-time asymptotic profile of the MLF is given by a viscous Burgers equation in which the viscosity parameter is proportional to $\Delta x^{2} / \Delta t$. Therefore, a smaller $\Delta t$ implies a higher numerical viscosity. Figure 17 highlights this pathology in the case of the gradient descent method.

The enhancement of the numerical oscillations on the recovered initial datum when reducing $\Delta t$ for MLF appears to be a purely linear issue. Actually, one can observe the same performance when solving the heat equation numerically backwards in time. Let us apply the same technique of the MLF to the heat equation:

$$
v_{t}-\nu v_{x x}=0 \approx \frac{v_{j}^{n+1}-\frac{v_{j-1}^{n}+2 v_{j}^{n}+v_{j+1}^{n}}{4}}{\Delta t}+\nu \frac{v_{j-1}^{n}-2 v_{j}^{n}+v_{j+1}^{n}}{\Delta x^{2}}=0 .
$$

In the Fourier space we have that

$$
\hat{v}(t, \xi)=\mathrm{e}^{-\nu t \xi^{2}} \hat{v}(0, \xi)
$$

and

$$
\hat{v}^{n}(\xi)=\left(1-\left(4 \nu \frac{\Delta t}{\Delta x^{2}}+1\right) \sin ^{2}\left(\frac{\xi \Delta x}{2}\right)\right)^{n} \hat{v}^{0}(\xi) .
$$

Hence, the inverse problem is clearly ill-posed, both from the continuous and the discrete points of view. In fact, we get, respectively,

$$
\hat{v}(0, \xi)=\mathrm{e}^{\nu T \xi^{2}} \hat{v}(T, \xi)
$$

and

$$
\hat{v}^{0}(\xi)=\left(1-\left(4 \nu \frac{\Delta t}{\Delta x^{2}}+1\right) \sin ^{2}\left(\frac{\xi \Delta x}{2}\right)\right)^{-N} \hat{v}^{N}(\xi) .
$$

Note that, of course, a final solution $v(x, T)$ in $L^{2}(\mathbb{R})$ does not guarantee that the initial data is in $L^{2}(\mathbb{R})$, since each frequency $\xi \in \mathbb{R}$ is weighted by an exponential term $\mathrm{e}^{\nu T \xi^{2}}$. Moreover, for the corresponding initial datum to be in $L^{2}(\mathbb{R})$, one needs the final target to be in an exponentially weighted space in the frequency domain.

The discrete case is even more sensitive. In fact, a Taylor expansion of the weights in (6.3) and (6.4) already denotes a substantial difference in the second term:

$$
\mathrm{e}^{\nu T \xi^{2}}=1+\nu T \xi^{2}+O\left(\xi^{4}\right),
$$



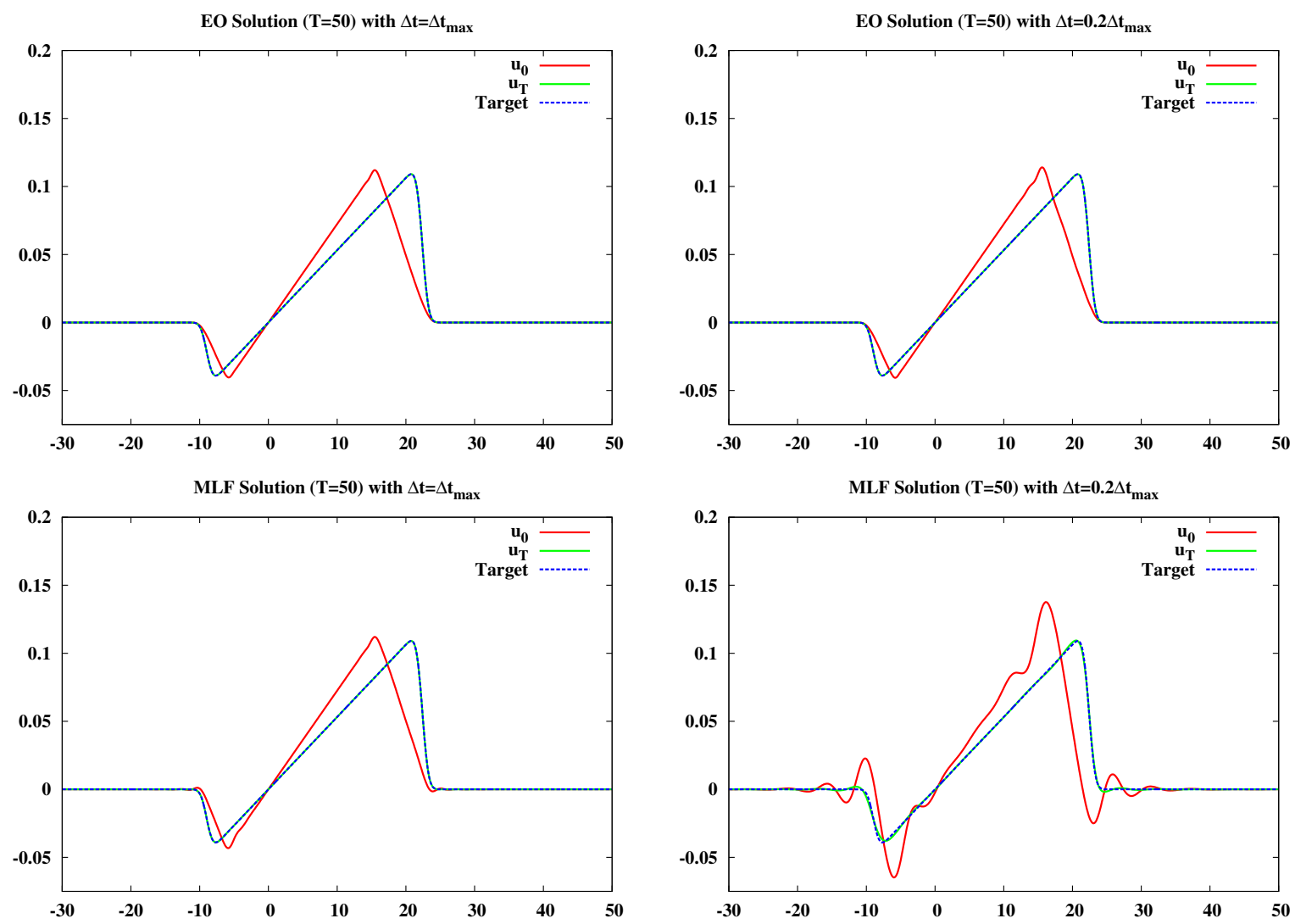

Figure 17. Initial data $u^{0}(x)$ for inviscid Burgers equation obtained after 300 iterations of the GDM+EO (top) and GDM+MLF (bottom). We use $\Delta x=0.1$ and $\Delta t=\Delta t_{\max }$ (left) and $\Delta t=0.2 \Delta t_{\max }($ right $)$.

while, taking $N=T / \Delta t$,

$$
\left(1-\left(4 \nu \frac{\Delta t}{\Delta x^{2}}+1\right) \sin ^{2}\left(\frac{\xi \Delta x}{2}\right)\right)^{-N}=1+\left(\frac{\Delta x^{2} T}{4 \Delta t}+\nu T\right) \xi^{2}+O\left(\xi^{4}\right) .
$$

Therefore, the dissipation is enhanced in the whole spectrum when making $\Delta t$ smaller for a fixed $\Delta x$.

\subsection{The set of reachable target functions}

The aim of the forthcoming discussion is to informally illustrate the underlying performance of the discrete approximations with less degree of rigor in the terminology.

Let us denote by $S(t)$ the semigroup corresponding to equation (1.1). The approximated solutions given by (3.1) generate a semigroup too. Let us denote by $S_{\Delta}^{E O}(t)$ the one corresponding to Engquist-Osher and by $S_{\Delta}^{M L F}(t)$ the one for modified Lax-Friedrichs. The previous experiments exhibit that $S_{\Delta}^{E O}(T)\left[L^{1}(\mathbb{R})\right]$ approximates quite accurately the set $S(T)\left[L^{1}(\mathbb{R})\right]$, even for large values of $T$. On the other hand, MLF requires smaller values of $\Delta x$ to reproduce $S(T)\left[L^{1}(\mathbb{R})\right]$ (see Fig. 18). For large enough $\Delta x$, MLF looks for the closest solution within $S_{\Delta}^{M L F}(T)\left[L^{1}(\mathbb{R})\right]$, producing the spurious oscillations that we have shown. 

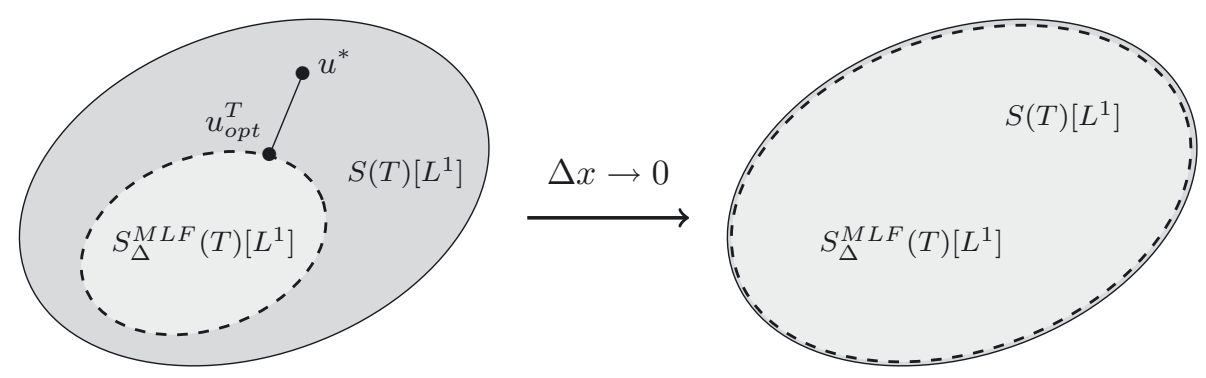

Figure 18. Set of functions that are reachable at time $T$ by the semigroup $S(t)$ of the Burgers equation for $L^{1}$ initial data and the semigroup $S_{\Delta}^{M L F}(t)$ associated to the modified Lax-Friedrichs scheme. As $\Delta x \rightarrow 0$, the set $S_{\Delta}^{M L F}(T)\left[L^{1}(\mathbb{R})\right]$ gets closer to $S(T)\left[L^{1}(\mathbb{R})\right]$.
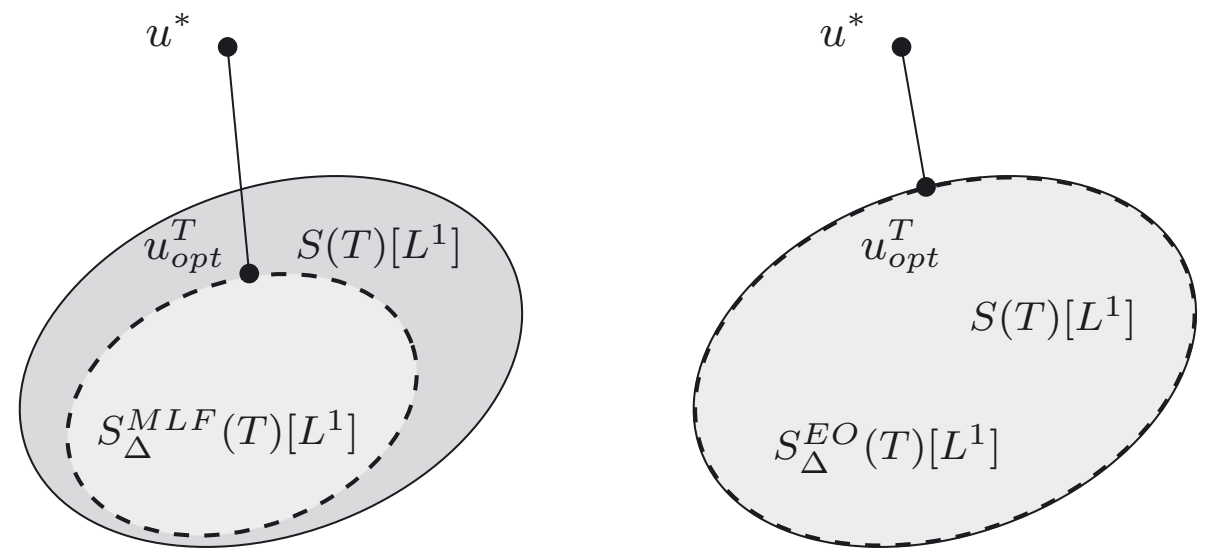

FIGURE 19. Projection of the target function onto the reachable sets by modified Lax-Friedrichs (left) and Engquist-Osher (right). The approximation obtained by EO is closer than the one by MLF.

In all previous experiments we have used target functions that can be very well approximated following the dynamics of the Burgers equation. In particular, we have chosen functions that satisfy the Oleinik condition. It is well known that solutions to (1.1) satisfy $u_{x} \leq 1 / t$ in the sense of distributions.

If the target is not in $S(T)\left[L^{1}(\mathbb{R})\right]$ (for instance, it is enough to take a function $u^{*}$ such that $u_{x}^{*}(T)>1 / T$ in some interval), both numerical semigroups will project the target onto their reachable sets (Fig. 19). Since the set $S_{\Delta}^{M L F}(T)\left[L^{1}(\mathbb{R})\right]$ is smaller than $S(T)\left[L^{1}(\mathbb{R})\right]$, MLF will make use of spurious oscillations again.

Let us illustrate this with another experiment. Let us consider a new target function $u^{* *}$, given by

$$
u^{* *}(x)= \begin{cases}\frac{1}{100}\left(-\mathrm{e}^{-(5 \sqrt{20}+x)^{2}}+\mathrm{e}^{-(2 \sqrt{20}+x)^{2}}\right. & \\ \quad+\sqrt{\pi} x(\operatorname{erf}(5 \sqrt{20}-x)+\operatorname{erf}(2 \sqrt{20}+x))), & |x-5| \leq 25, \\ 0, & \text { elsewhere }\end{cases}
$$

Note that $u^{* *}$ is the same as $u^{*}$, but with a larger amplitude. In this case, we have chosen the target function such that $u_{x}^{* *}(x)>1 / 50$ in some interval. Therefore, $u^{* *}$ cannot be reached at $T=50$ following the dynamics of the Burgers equation, not even approximately. 

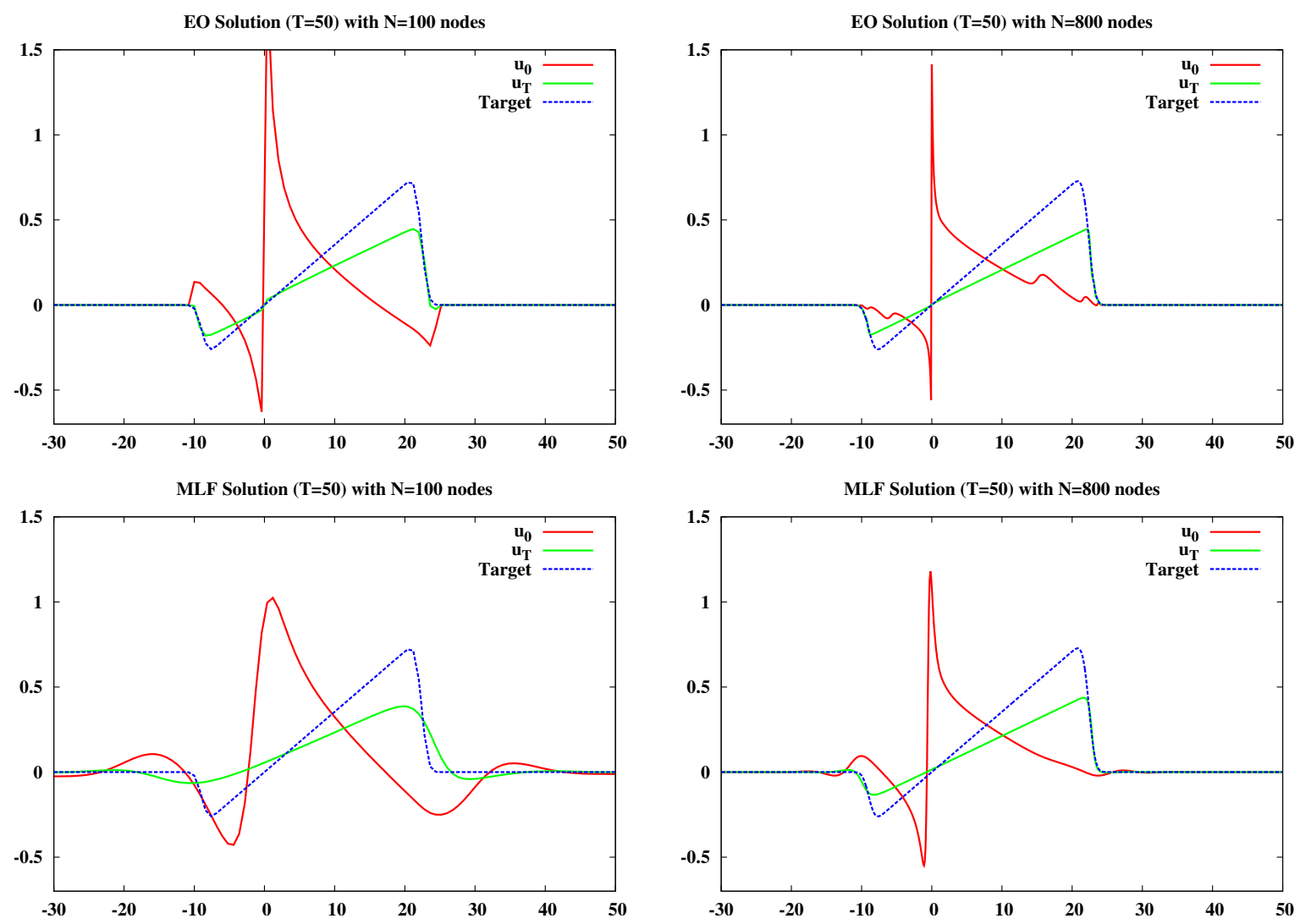

Figure 20. Initial data $u^{0}(x)$ obtained using GDM+EO (top) and GDM+MLF (bottom) to solve (3.1)-(3.7) for the target function $u^{* *}$. We use $\Delta x=0.8$ (left) and $\Delta x=0.1$ (right) with $\nu=10^{-4}$.

Indeed, we can observe in Figure 20 that the optimal solutions do not fit $u^{* *}$. Let us remark that the closest function that GDM can recover at final time $T=50$ is precisely the one that has slope of $1 / 50$ in the intermediate part which is the maximum slope allowed by the Oleinik condition.

Let us reinterpret the situation from a different point of view. Assume we are given a time $T$ in which $u^{* *}$ can be fully reached. The way the GDM tries to recover $u^{* *}$ is by moving both peaks back towards $x=0$ and slightly increasing their amplitude. As the final time $T$ is increased, the peaks will be moved further to $x=0$. There exists a maximum finite time this can be done before forcing the initial data to be multivalued. The maximum time is inversely proportional to $\left\|u_{x}^{* *}\right\|_{\infty}$, according to the Oleinik condition. When dealing with $T$ larger than this maximum time allowed, GDM follows the same procedure but from the closest achievable function which satisfies $u_{x} \leq 1 / T$, as we show in the simulations.

\section{Functional LANDSCAPES}

In this section we plot some of the landscapes of the functionals we have minimized, in order to explain the observed phenomena in the behavior of the minimizers. As discussed in Section 4, the obtained minimizers exhibit a critical sensitivity to the choice of EO, or MLF in large-time behavior regime.

The difference among the obtained solutions could correspond to the presence of multiple local minimizers of the functionals under consideration. In order to shed light on this issue a simple experiment is proposed to 

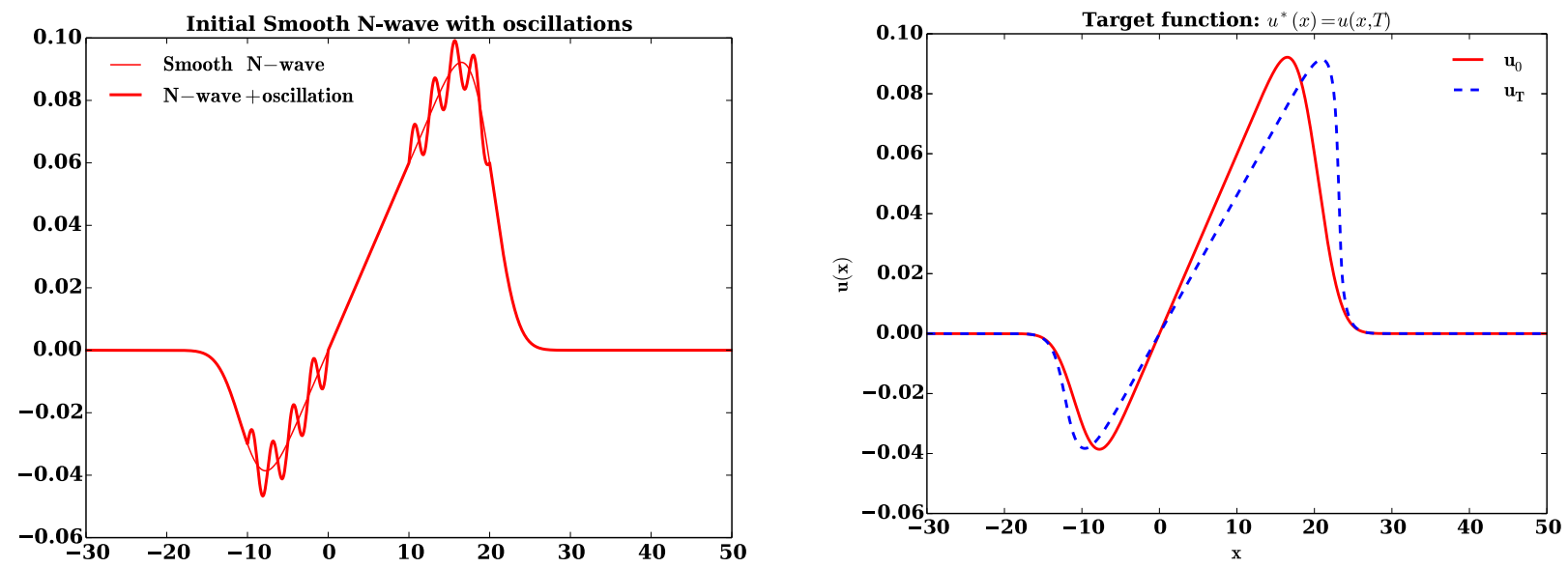

FiguRE 21. Initial conditions are constructed from superposing parameterized sinusoidal oscillations on a base "smooth N-wave" (left). Target function $u^{*}$ is constructed from the evolution of the initial data corresponding to the $\mathrm{N}$-wave and free from any oscillations. The optimal solution would be a N-wave without superposed oscillations. Final time is $T=50$ and physical viscosity is $\nu=10^{-4}$.

visualize the functional $\mathcal{J}\left(u_{0}\right)$ for a set of initial conditions parameterized with two degrees of freedom. The functional landscape is plotted as these parameters vary, to check the possible presence of multiple wells.

For constructing the initial data, a set of parameterized sinusoidal waves are superposed on a smooth $\mathrm{N}$-wave shaped function $w$, obtained by subtracting and scaling two error functions. The oscillatory signals are placed only in the neighborhood of sharp slopes of the $\mathrm{N}$-wave and they are parameterized with their amplitude $r$ and frequency $s$ as follows:

$$
u_{r, s}^{0}(x)= \begin{cases}w(x)+r \sin (s x), & \text { near steep fronts } \\ w(x), & \text { elsewhere }\end{cases}
$$

where

$$
w(x)=h x(\operatorname{erf}(m(x-a))-\operatorname{erf}(m(x-b))
$$

Parameters $a$ and $b$ are related to the location of the peaks and $m$ to the sharp slopes they create. Also, the slope of the middle part of the $\mathrm{N}$-wave is proportional to $h$. In any case, we will fix all of them, leaving $r$ and $s$ the only degrees of freedom. A sample initial condition with sinusoidal oscillations is depicted in Figure 21(left).

The target function that we choose in this case is selected such that it corresponds to the solution of viscous Burgers equation at $t=T$ starting from $u_{0,0}^{0}$; this means that the desired initial condition does not contain any sinusoidal oscillation. Hence, optimal solutions will be achieved for all pairs of $(r, 0)$, and $(0, s)$ where $r, s \in \mathbb{R}$ (which, indeed, correspond to the same function). Of course, the cost function value corresponding to these pairs would be exactly zero. A plot of the target function and its corresponding initial condition is shown in Figure 21(right).

In our experiment, we first sample pairs of $(r, s) \in[-0.025,0.025] \times[0,2 \pi]$. Then, we compute $u_{\Delta}(x, T)$ using (3.1) (with $\nu=10^{-4}$ ) from initial condition $u_{r, s}^{0}(x)$ and evaluate the corresponding cost function $\mathcal{J}_{\Delta}\left(u_{r, s}^{0}\right)$. In particular, we set $w$ with $a=-10, b=20, h=0.003$ and $m=0.3$; that is,

$$
w(x)=0.003 x(\operatorname{erf}(0.3(x+10))-\operatorname{erf}(0.3(x-20))
$$



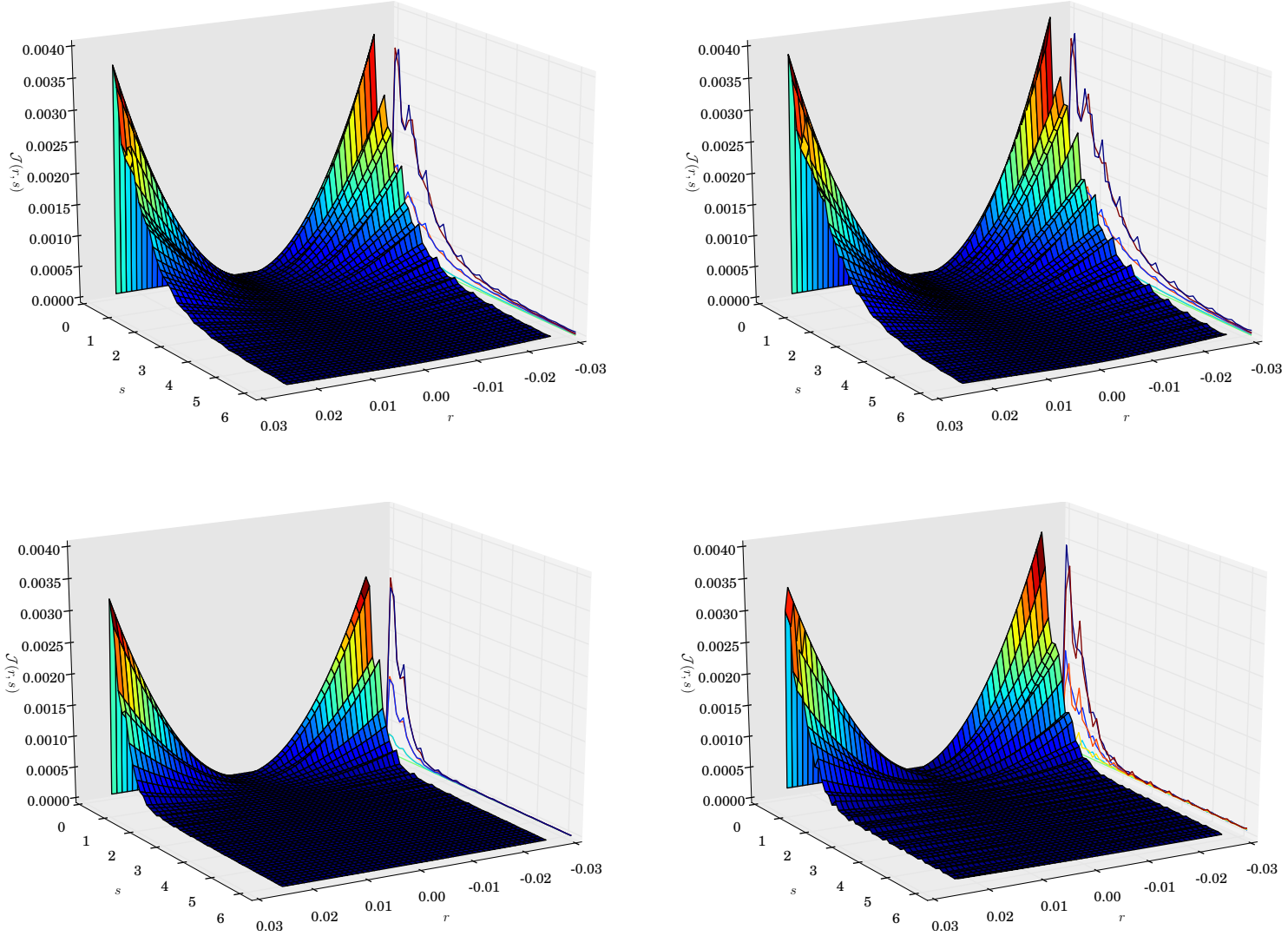

Figure 22. Functional landscape $(r, s, \mathcal{J})$; Engquist-Osher (top row) and modified Lax-Friedrichs (bottom row) fluxes are used for discretizing the viscous Burgers equation with $\Delta x=0.2$ (left), and $\Delta x=0.10$ (right). The landscape of MLF is less sensitive to the variation of parameters $(r, s)$ due the dissipative nature of MLF.

The surface plots of the cost function $\mathcal{J}\left(u_{r, s}^{0}\right)$ for the sampled $(r, s)$ are shown in Figure 22. In the top row, $u_{\Delta}$ is calculated using EO flux with $\Delta x=0.2$ (left), and $\Delta x=0.1$ (right) mesh-sizes; while in the bottom row, $u_{\Delta}$ is evaluated by using MLF flux.

As observed from figures, for the range of initial conditions considered, the functional landscapes obtained from EO and MLF discretizations show no presence of multiple wells. The landscape obtained from EO shows more sensitivity to the variation of the parameters. On the contrary, MLF dissipates sinusoidal signals and the landscape obtained from MLF is less sensitive to higher frequencies $s \gg 1$. Moreover, in the case of MLF the choice of $\Delta x$ (and, $\Delta t$ ) also impacts the sensitivity of the landscape.

\section{Conclusions AND PERspectives}

This paper further builds on the previous work done on analyzing discretization schemes that preserve the large-time behavior [26]. Here, we showed some of the pathologies that may arise in numerical approximation of optimal control problems in such regimes. In particular, we highlighted the performance of classical flux discretization methods and the consequences if the discretization scheme is not chosen properly. It was shown how the choice of the discretization scheme can alter the underlying dynamics of the system and its corresponding 
functional. Moreover, the optimal initial datum obtained by one discretization scheme may not be the best fit for the functional if it is evolved in time with a different numerical scheme.

In this regard, we presented several numerical experiments based on the resolution of the Burgers equation (both viscous and inviscid) for large times. We emphasize that, in large-time regime, the effect of numerical viscosity is pronounced. The optimization was done employing gradient descent method (GDM) and an open source interior point optimization software (IPOPT), but the conlusions can be extended to other optimization techniques.

In summary, following conclusions are made:

- The performance of MLF is not satisfactory in comparison with EO. The initial data obtained with MLF can be quite oscillatory in the vicinity of sharp slopes for course grids. Also, the convergence rate of MLF is slower in comparison with EO for similar grids.

- The performance of EO when employed in GDM or IPOPT is satisfactory. The initial data obtained is less sensitive to the choice of $\Delta x$ and $\Delta t$. Furthermore, the results are also less sensitive to the initial setting of the optimization algorithms.

- The numerical viscosity present in MLF is proportional to $\Delta x^{2} / \Delta t$; hence, the solution is quite dependent on the domain discretization. While coarse discretizations result in more numerical viscosity, small time steps can increase the numerical viscosity too. This point underlines the importance of selecting a proper CFL number in the simulations done with MLF.

- Results obtained from IPOPT with MLF were more oscillatory in comparison with those of GDM method. The MLF functional shows insensitivity to the high frequency oscillations as it was illustrated in the functional landscape. It is likely to obtain two dissimilar solutions in terms of high frequency contents if we slightly perturb the convergence tolerance in the optimization algorithm. This observation is consistent with the ill-posedness of the backward solution of the diffusion process.

- The possibility of presence of multiple distinct local minima in the functional landscape, when the target is approximately reachable, is excluded based on the functional landscape visualization. However, the functional landscape shows flatness (or lack of sensitivity) in the neighborhood of minimizers in the case of MLF.

- For optimization problems with physically unreachable target functions, which they do not satisfy Oleinik inequality, the optimization solvers try to fit the target as best as possible within their reachable sets. In these situations, EO performance is again more robust compared with MLF.

Acknowledgements. The authors thank the reviewers for their valuable comments and suggestions. This work is supported by the Advanced Grant FP7-246775 NUMERIWAVES of the European Research Council Executive Agency, FA955014-1-0214 of the EOARD-AFOSR, FA9550-15-1-0027 of AFOSR, the MTM2011-29306 and MTM2014-52347 Grants of the MINECO and a Humboldt Award at the University of Erlangen-Nürnberg. A. Pozo is also supported by the Basque Government (PREDOC Program 2012, 2013 and 2014).

\section{REFERENCES}

[1] P.-A. Absil, R. Mahony and B. Andrews, Convergence of the iterates of descent methods for analytic functions. SIAM J. Optim. 16 (2005) 531-547.

[2] J.J. Alonso and M.R. Colonno, Multidisciplinary optimization with applications to sonic-boom minimization. Ann. Rev. Fluid Mech. 44 (2012) 505-526.

[3] H. Attouch, J. Bolte and B.F. Svaiter, Convergence of descent methods for semi-algebraic and tame problems: proximal algorithms, forward-backward splitting, and regularized Gauss-Seidel methods. Math. Progr. 137 (2013) 91-129.

[4] C. Bardos and O. Pironneau, Data assimilation for conservation laws. Methods Appl. Anal. 12 (2005) $103-134$.

[5] F. Bouchut and F. James, One-dimensional transport equations with discontinuous coefficients. Nonlinear Anal.: Theory, Methods Appl. 32 (1998) 891-933.

[6] F. Bouchut and F. James, Differentiability with respect to initial data for a scalar conservation law. In Hyperbolic Problems: Theory, Numerics, Applications, edited by M. Fey and R. Jeltsch. Vol. 129 of International Series of Numerical Mathematics. Springer-Verlag (1999) 113-118.

[7] Y. Brenier and S. Osher, The discrete one-sided Lipschitz condition for convex scalar conservation laws. SIAM J. Numerical Anal. 25 (1988) 8-23. 
[8] A. Bressan and A. Marson, A variational calculus for discontinuous solutions of systems of conservation laws. Commun. Partial Differ. Eq. 20 (1995) 1491-1552.

[9] C. Castro, F. Palacios and E. Zuazua, An alternating descent method for the optimal control of the inviscid Burgers' equation in the presence of shocks. Math. Models Methods Appl. Sci. 18 (2008) 369-416.

[10] C. Castro, F. Palacios and E. Zuazua, Optimal control and vanishing viscosity for the Burgers equation. In Chapter 7 of Integral Methods in Science and Engineering, edited by C. Costanda and M.E. Pérez. Birkhäuser Verlag 2 (2010) 65-90.

[11] Ph.G. Ciarlet, Introduction to numerical linear algebra and optimisation. Vol. 2 of Cambridge Texts in Applied Mathematics. Cambridge University Press (1989).

[12] R.O. Cleveland, Propagation of sonic booms through a real, stratified atmosphere. Ph.D. thesis, University of Texas at Austin (1995).

[13] S. Ervedoza and E. Zuazua, Numerical Approximation of Exact Controls for Waves. Springer Briefs in Mathematics. SpringerVerlag (2013).

[14] E. Fernández-Cara and E. Zuazua, The cost of approximate controllability for heat equations: The linear case. Adv. Differ. Equ. 5 (2000) 465-514.

[15] R. Fourer, D.M. Gay and B.W. Kernighan, A modeling language for mathematical programming. Manag. Sci. 36 (1990) $519-554$.

[16] M. Ghil and P. Malanotte-Rizzoli, Data assimilation in meteorology and oceanography. Vol. 33 of Advances in Geophysics. Academic Press (1991).

[17] M.B. Giles, Discrete adjoint approximations with shocks. In Hyperbolic Problems: Theory, Numerics, Applications, edited by Th.Y. Hou and E. Tadmor. Springer-Verlag (2003) 185-194.

[18] M.B. Giles and N.A. Pierce, An introduction to the adjoint approach to design. Turbulence and Combustion 65 (2000) $393-415$.

[19] M.B. Giles and S. Ulbrich, Convergence of linearized and adjoint approximations for discontinuous solutions of conservation laws. Part 1: linearized approximations and linearized output functionals. SIAM J. Numer. Anal. 48 (2010) 882-904.

[20] M.B. Giles and S. Ulbrich, Convergence of linearized and adjoint approximations for discontinuous solutions of conservation laws. Part 2: adjoint approximations and extensions. SIAM J. Numer. Anal. 48 (2010) 905-921.

[21] R. Glowinski, J.-Louis Lions and J. He, Exact and Approximate Controllability for Distributed Parameter Systems: A Numerical Approach. Vol. 117 of Encyclopedia of Mathematics and its Applications. Cambridge University Press (2008).

[22] E. Godlewski and P.-A. Raviart, Hyperbolic systems of conservation laws. Number 3 in Mathematiques \& Applications. Ellipses (1991).

[23] L. Gosse and F. James, Numerical approximations of one-dimensional linear conservation equations with discontinuous coefficients. Math. Comput. 69 (2000) 987-1015.

[24] A. Haraux, Some applications of the Łojasiewicz gradient inequality. Commun. Pure Appl. Anal. 11 (2012) $2417-2427$.

[25] E. Hopf, The partial differential equation $u_{t}+u u_{x}=\mu u_{x x}$. Commun. Pure Appl. Math. 3 (1950) 201-230.

[26] L.I. Ignat, A. Pozo and E. Zuazua, Large-time asymptotics, vanishing viscosity and numerics for 1-D scalar conservation laws. Math. Comput. 84 (2015) 1633-1662.

[27] F. James and M. Sepúlveda, Convergence results for the flux identification in a scalar conservation law. SIAM J. Control Optim. 37 (1999) 869-891.

[28] F. James and N. Vauchelet, A remark on duality solutions for some weakly nonlinear scalar conservation laws. C. R. Acad. Sci. 349 (2011) 657-661.

[29] Y.-J. Kim and A.E. Tzavaras, Diffusive N-waves and metastability in the Burgers equation. SIAM J. Math. Anal. 33 (2001) 607-633.

[30] Y. Li, S. Osher and R. Tsai, Heat source identification based on $l_{1}$ constrained minimization. Inverse Probl. Imaging 8 (2014) 199-221.

[31] J.-Louis Lions and B. Malgrange, Sur l'unicité rétrograde dans les problèmes mixtes paraboliques. Math. Scand. 8 (1960) $277-286$.

[32] T.-P. Liu and M. Pierre, Source-solutions and asymptotic behavior in conservation laws. J. Diff. Equ. 51 (1984) $419-441$.

[33] B. Merlet and M. Pierre, Convergence to equilibrium for the backward Euler scheme and applications. Commun. Pure Appl. Anal. 8 (2010) 685-702.

[34] J. Nocedal and S.J. Wright, Numerical Optimization. Springer Series in Operations Research and Financial Engineering. 2nd edition. Springer-Verlag (2006).

[35] S. Ulbrich, Optimal control of nonlinear hyperbolic conservation laws with source terms. Habilitation Thesis, Fakultät für Mathematik, Technische Universität München (2001).

[36] S. Ulbrich, A sensitivity and adjoint calculus for discontinuous solutions of hyperbolic conservation laws with source terms. SIAM J. Control Optim. 41 (2002) 740-797.

[37] A. Wächter and L.T. Biegler, On the implementation of an interior-point filter line-search algorithm for large-scale nonlinear programming. Math. Progr. 106 (2006) 25-57.

[38] G.B. Whitham, Linear and nonlinear waves. John Wiley \& Sons (1974).

[39] E. Zuazua, Propagation, observation, and control of waves approximated by finite difference methods. SIAM Rev. 47 (2005) 197-243. 